\title{
On Parametric Gevrey Asymptotics for a $q$-Analog of Some Linear Initial Value Problem
}

\author{
By \\ Stéphane MALEK \\ (University of Lille 1, France)
}

\begin{abstract}
We study an inhomogeneous linear $q$-difference differential Cauchy problem, with a complex perturbation parameter $\varepsilon$, whose coefficients depend holomorphically on $\varepsilon$ and on time in the vicinity of the origin in $C^{2}$ and are bounded analytic on some horizontal strip in $\boldsymbol{C}$ w.r.t the space variable. This problem is seen as a $q$-analog of an initial value problem recently investigated by the author and A. Lastra in [9]. Here a comparable result with the one in [9] is achieved, namely we construct a finite set of holomorphic solutions on a common bounded open sector in time at the origin, on the given strip above in space, when $\varepsilon$ belongs to a well selected set of open bounded sectors whose union covers a neighborhood of 0 in $\boldsymbol{C}^{*}$. These solutions are constructed through a continuous version of a $q$-Laplace transform of some order $k \geq 1$ introduced newly in [6] and Fourier inverse map of some function with $q$-exponential growth of order $k$ on adequate unbounded sectors in $\boldsymbol{C}$ and with exponential decay in the Fourier variable. Moreover, by means of a $q$-analog of the classical Ramis-Sibuya theorem, we prove that they share a common formal power series (that generally diverge) in $\varepsilon$ as $q$-Gevrey asymptotic expansion of order $1 / k$.

Key Words and Phrases. $q$-Laplace transform, $q$-Borel transform, Perturbative expansion, $q$-Gevrey asymptotic expansion, Formal power series.
\end{abstract}

2010 Mathematics Subject Classification Numbers. 35C10, 35C20.

\section{Introduction}

The work of this paper follows in the footsteps of a series of results dedicated to the asymptotic study for holomorphic solutions to different kind of $q$-difference-differential Cauchy problems involving so-called irregular singularities investigated by the author and co-authors these recent years, see [8], [10], [11], [12]. Recently, in this framework of mixed type equations, another approach was highlighted by $\mathrm{H}$. Tahara and $\mathrm{H}$. Yamazawa for the construction of a $q$-analog of summability for formal solutions to inhomogeneous linear $q$-difference-differential which leans on Newton polygon procedures used in the context of partial differential equations by S. Ouchi, see [19]. We mention also the novel contribution of $\mathrm{H}$. Yamazawa dealing with nonlinear $q$-analogs of Briot-Bouquet type PDE, [21]. These contributions take root in 
the seminal analytic studies of linear meromorphic $q$-difference systems of the form

$$
Y(q z)=A(z) Y(z)
$$

for $q \in C$ with $|q|>1$ performed by J-P. Ramis, J. Sauloy, C. Zhang et al. see for instance [4], [5], [6], [14], [15], [16], [17], [18], [22]. Our main result will bring into play analytic tools such as $q$-analogs of the classical BorelLaplace transforms of some positive order $k>0$ put forward by C. Zhang and F. Marotte in their resummation theory of formal power series solutions to $q$-difference equations, see [14], used more lately by L. Divizio and C. Zhang for confluence problems in [5] and by $T$. Dreyfus in his construction of meromorphic solutions to $q$-difference equations as iterated discrete $q$-Laplace transforms in [6].

In this work, we consider the following parameter depending inhomogeneous linear Cauchy problems with the shape

$$
Q\left(\partial_{z}\right) \sigma_{q, t} u(t, z, \varepsilon)=\sum_{l=1}^{D} \varepsilon^{\Delta_{l}} t^{d_{l}} \sigma_{q, t}^{\delta_{l}}\left(c_{l}(t, z, \varepsilon) R_{l}\left(\partial_{z}\right) u(t, z, \varepsilon)\right)+f(t, z, \varepsilon),
$$

where $D \geq 3, \Delta_{l}, d_{l}, 1 \leq l \leq D$ are non negative integers, $\delta_{l} \geq 1$ are rational numbers or integers, $Q(X), R_{l}(X)$ are polynomials with complex coefficients and where the coefficients $c_{l}(t, z, \varepsilon)$ and the forcing term $f(t, z, \varepsilon)$ define bounded holomorphic functions near the origin in $C^{2}$ w.r.t $(t, \varepsilon)$ and on a horizontal strip $H_{\beta}=\{z \in C|| \operatorname{Im}(z) \mid<\beta\}$ of width $\beta>0$ w.r.t the space variable $z$. The problem (1) involves $q$-difference operators $\sigma_{q, t}^{\gamma}$ acting on the $t$ variable in the form $t \mapsto t q^{\gamma}$ where $q>1$ is some positive real number, $\gamma$ are rational numbers and derivations with respect to $z$. The equation (1) under study can be seen as a $q$-analog of the initial value problem investigated in our previous work [9] in its linear version

$$
\begin{aligned}
\mathbf{Q}\left(\partial_{z}\right)\left(\partial_{t} y(t, z, \varepsilon)\right)= & \sum_{l=1}^{\mathbf{D}} \varepsilon^{\boldsymbol{A}_{l}} t^{\mathbf{d}_{l}} \partial_{t}^{\boldsymbol{\delta}_{l}} R_{l}\left(\partial_{z}\right) y(t, z, \varepsilon) \\
& +\mathbf{c}_{0}(t, z, \varepsilon) \mathbf{R}_{0}\left(\partial_{z}\right) y(t, z, \varepsilon)+\mathbf{f}(t, z, \varepsilon)
\end{aligned}
$$

for given vanishing initial data $y(0, z, \varepsilon) \equiv 0$, well chosen integers $\mathbf{D} \geq 2, \boldsymbol{\Delta}_{l}$, $\mathbf{d}_{l}, \boldsymbol{\delta}_{l} \geq 0,1 \leq l \leq \mathbf{D}$ and where $\mathbf{Q}(X), \mathbf{R}_{l}(X), 0 \leq l \leq \mathbf{D}$ are complex valued polynomials and the coefficient $\mathbf{c}_{0}(t, z, \varepsilon)$ together with the forcing term $\mathbf{f}(t, z, \varepsilon)$ are holomorphic and bounded as in the main problem (1) mentioned above. The problem (1) is a discretized version of (2) in the sense that the derivative $\partial_{t}$ is replaced by the operator $(f(q t)-f(t)) /(q t-t)$ for $q>1$ (which formally tends to $\partial_{t}$ as $q$ tends to 1). In [9], for a given suitable set of open bounded sectors $\left\{\mathcal{E}_{p}\right\}_{0 \leq p \leq \varsigma-1}$ whose union covers a full neighborhood of 0 in $\boldsymbol{C}^{*}$, for 
some integer $\varsigma \geq 2$ and for well selected directions $\mu_{p} \in \boldsymbol{R}$, one constructs a family of holomorphic bounded functions $y_{p}(t, z, \varepsilon)$, solutions of (2) on products $\mathcal{T} \times H_{\beta} \times \mathcal{E}_{p}$, where $\mathcal{T}$ stands for a fixed bounded sector centered at 0 with small aperture, that can be written as Laplace transforms of some adequate order $\mathbf{k} \geq 1$ in direction $\mu_{p}$ and Fourier inverse transform

$$
y_{p}(t, z, \varepsilon)=\frac{\mathbf{k}}{(2 \pi)^{1 / 2}} \int_{-\infty}^{+\infty} \int_{L_{\mu_{p}}} \omega_{p}(u, m, \varepsilon) e^{-(u /(\varepsilon t))^{\mathbf{k}}} e^{i z m} \frac{d u}{u} d m,
$$

where the inner integration is made along some halfline $L_{\mu_{p}}=\boldsymbol{R}_{+} e^{\sqrt{-1}} \mu_{p}$ and where $\omega_{p}(u, m, \varepsilon)$ denotes a function with at most exponential growth of order $\mathbf{k}$ in $u / \varepsilon$ and with exponential decay in $m \in \boldsymbol{R}$. Moreover, all these functions $y_{p}(t, z, \varepsilon)$ turn out to share a common formal power series $\hat{y}(t, z, \varepsilon)=$ $\sum_{m \geq 0} g_{m}(t, z) \varepsilon^{m} \in \boldsymbol{G}[[\varepsilon]]$, where $\boldsymbol{G}$ is the Banach space of bounded holomorphic functions on $\mathcal{T} \times H_{\beta}$ endowed with the $L_{\infty}$ norm, as asymptotic expansion of Gevrey order $1 / \mathbf{k}$ on $\mathcal{E}_{p}$.

In this work, we will explain and present a similar result for the problem (1). Namely, we build a set of actual holomorphic solutions $u_{p}(t, z, \varepsilon)$ to the problem (1) on domains $\mathcal{T} \times H_{\beta} \times \mathcal{E}_{p}$ similar to the ones mentioned above, which can be expressed as a continuous version of a $q$-analog of a Laplace transform described in [6] of a well chosen order $k \geq 1$ and Fourier inverse transform

$$
u_{p}(t, z, \varepsilon)=\frac{1}{(2 \pi)^{1 / 2}} \int_{-\infty}^{+\infty} \int_{L_{\gamma_{p}}} \frac{\omega_{k}(u, m, \varepsilon)}{\Theta_{q^{1 / k}}(u /(\varepsilon t))} e^{i z m} \frac{d u}{u} d m,
$$

where $\Theta_{q^{1 / k}}(x)$ stands for the Jacobi Theta function of order $k$ given by its Laurent expansion (34), the inner integration is made along a halfline $L_{\gamma_{p}}=$ $\boldsymbol{R}_{+} e^{\sqrt{-1} \gamma_{p}}$ and where $w_{k}(u, m, \varepsilon)$ represents a function with at most $q$-exponential growth of order $k$ w.r.t $u$ on unbounded sectors, exponential decay w.r.t $m$ on $\boldsymbol{R}$ and depends analytically on $\varepsilon$ near 0 . Furthermore, these functions $u_{p}, 0 \leq$ $p \leq \varsigma-1$, possess a common formal power series $\hat{u}(t, z, \varepsilon)=\sum_{m \geq 0} h_{m}(t, z) \varepsilon^{m} \in$ $\boldsymbol{G}[[\varepsilon]]$, where $\boldsymbol{G}$ stands, as above, for the Banach space of bounded holomorphic functions on $\mathcal{T} \times H_{\beta}$ with $L_{\infty}$ norm, as $q$-Gevrey asymptotic expansion of order $1 / k$ on $\mathcal{E}_{p}$, meaning that for any closed sector $\mathcal{W} \subset \mathcal{E}_{p}$ centered at 0 , there exist two constants $C, M>0$ with

$$
\sup _{t \in \mathcal{T}, z \in H_{\beta}}\left|u_{p}(t, z, \varepsilon)-\sum_{m=0}^{n} h_{m}(t, z) \varepsilon^{m}\right| \leq C M^{n+1} q^{n(n+1) /(2 k)}|\varepsilon|^{n+1}
$$

for all $n \geq 0$, all $\varepsilon \in \mathcal{W}$.

The paper is organized as follows. In Section 2, we introduce some weighted Banach spaces of continuous functions with $q$-exponential growth on unbounded sectors in $\boldsymbol{C}$ and with exponential decay on $\boldsymbol{R}$. We study the 
continuity of $q$-difference operators with polynomial coefficients and $q$-analogs of the convolution of order $k$ acting on these spaces. In Section 3, we recall the definition of the $q$-Borel transform of order $k$ and we provide commutation formulas w.r.t products and $q$-differential operators with polynomial coefficients. We state also several analytic properties of a $q$-Laplace transform of order $k$. In Section 4 , we introduce an auxiliary $q$-difference and convolution problem for which we supply a formal solution. We show that its $q$-Borel transform of order $k$ satisfies a convolution equation. Under appropriate constraints, one can solve uniquely this latter equation within the Banach spaces described in Section 2 with the help of some fixed point theorem argument. Applying a $q$-Laplace transform, we can settle an actual solution of the former auxiliary problem having the given formal solution as $q$-Gevrey asymptotic expansion. In Section 5, using the construction of Section 4, we can provide a set of actual holomorphic solutions to our initial Cauchy problem (1) on bounded sectors w.r.t the perturbation parameter $\varepsilon$. We show that the difference of any two neighboring solutions tends to 0 as $\varepsilon$ tends to 0 faster than a function with $q$-exponential decay of order $k$ (Theorem 1). In Section 6, we show the existence of a common asymptotic expansion of $q$-Gevrey order $1 / k$ for these actual solutions as $\varepsilon$ goes to 0 on sectors (Theorem 2). The result leans on a $q$-analog of the classical Ramis-Sibuya theorem introduced in a recent contribution of A. Lastra and the author in [10].

\section{Some Banach spaces of functions}

\subsection{Banach spaces of functions with $q$-exponential growth and exponential decay}

Let $S_{d}$ be an open unbounded sector with bisecting direction $d \in \boldsymbol{R}$ centered at 0 in $\boldsymbol{C}$. By convention, all the sectors considered in this paper do not contain the origin in $C$.

Definition 1. Let $k, \beta, \mu>0, q>1$ and $\alpha$ be real numbers. We denote $\operatorname{Exp}_{(k, \beta, \mu, \alpha)}^{q}$ the vector space of complex valued continuous functions $(\tau, m) \mapsto$ $h(\tau, m)$ on $\bar{S}_{d} \times \boldsymbol{R}$, which are holomorphic with respect to $\tau$ on $S_{d}$ and such that

$\|h(\tau, m)\|_{(k, \beta, \mu, \alpha)}=\sup _{\tau \in \bar{S}_{d}, m \in \boldsymbol{R}}(1+|m|)^{\mu} e^{\beta|m|} \exp \left(-\frac{k}{2} \frac{\log ^{2}(|\tau|)}{\log (q)}-\alpha \log (|\tau|)\right)|h(\tau, m)|$

is finite. One can check that the normed space $\left(\operatorname{Exp}_{(k, \beta, \mu, \alpha)}^{q},\|\cdot\|_{(k, \beta, \mu, \alpha)}\right)$ is a Banach space.

Remark. These norms are built in order that all entire functions $h(\tau)$ on $\boldsymbol{C}$ with $q$-exponential growth of order $k$ studied in the work [15] by J-P. Ramis 
belong to these spaces. Notice that similar norms have already been used by A. Lastra and the author in a previous joint work [8].

Throughout the whole section, we assume $k, \beta, \mu>0$ and $\alpha$ are fixed. In the next lemma, we check the continuity property for the multiplication operation with bounded functions.

Lemma 1. Let $(\tau, m) \mapsto a(\tau, m)$ be a bounded continuous function on $\bar{S}_{d} \times \boldsymbol{R}$, which is holomorphic with respect to $\tau$ on $S_{d}$. Then, we have

$$
\|a(\tau, m) h(\tau, m)\|_{(k, \beta, \mu, \alpha)} \leq\left(\sup _{\tau \in \bar{S}_{d}, m \in \boldsymbol{R}}|a(\tau, m)|\right)\|h(\tau, m)\|_{(k, \beta, \mu, \alpha)}
$$

for all $h(\tau, m) \in \operatorname{Exp}_{(k, \beta, \mu, \alpha)}^{q}$.

In the next proposition, we study the continuity property for the multiplication operation by functions with at most polynomial growth and contraction operators acting on the latter Banach spaces. For any real number $\gamma \geq 0$, the contraction operator $\sigma_{q, \tau}^{-\gamma}$ is defined as $\sigma_{q, \tau}^{-\gamma} h(\tau, m)=h\left(\tau / q^{\gamma}, m\right)$ for any complex valued function $h$ on $\bar{S}_{d} \times \boldsymbol{R}$.

Proposition 1. Let $\gamma_{1}, \gamma_{2}, \gamma_{3} \geq 0$ be real numbers such that

$$
\gamma_{2} \geq k \gamma_{3}, \quad \gamma_{1}+k \gamma_{3} \geq \gamma_{2} \text {. }
$$

Let $a_{\gamma_{1}}(\tau)$ be a holomorphic function on $S_{d}$, continuous on $\bar{S}_{d}$ with

$$
\left|a_{\gamma_{1}}(\tau)\right| \leq \frac{1}{(1+|\tau|)^{\gamma_{1}}}
$$

for all $\tau \in \bar{S}_{d}$. Then, there exists a constant $C_{1}>0$ (depending on $k, q, \alpha, \gamma_{1}$, $\left.\gamma_{2}, \gamma_{3}\right)$ with

$$
\left\|a_{\gamma_{1}}(\tau) \tau^{\gamma_{2}} \sigma_{q, \tau}^{-\gamma_{3}} f(\tau, m)\right\|_{(k, \beta, \mu, \alpha)} \leq C_{1}\|f(\tau, m)\|_{(k, \beta, \mu, \alpha)}
$$

for all $f(\tau, m) \in \operatorname{Exp}_{(k, \beta, \mu, \alpha)}^{q}$.

Proof. Let $f(\tau, m) \in \operatorname{Exp}_{(k, \beta, \mu, \alpha)}^{q}$. By definition, we can write

$$
\begin{aligned}
\left\|a_{\gamma_{1}}(\tau) \tau^{\gamma_{2}} \sigma_{q, \tau}^{-\gamma_{3}} f(\tau, m)\right\|_{(k, \beta, \mu, \alpha)} \\
=\sup _{\tau \in \bar{S}_{d}, m \in \boldsymbol{R}}(1+|m|)^{\mu} e^{\beta|m|} \exp \left(-\frac{k}{2} \frac{\log ^{2}(|\tau|)}{\log (q)}-\alpha \log (|\tau|)\right) \\
\quad \times\left|a_{\gamma_{1}}(\tau) \tau^{\gamma_{2}}\right|\left|f\left(\tau / q^{\gamma_{3}}, m\right)\right| \exp \left(-\frac{k}{2} \frac{\log ^{2}\left(\left|\tau / q^{\gamma_{3}}\right|\right)}{\log (q)}-\alpha \log \left(\left|\tau / q^{\gamma_{3}}\right|\right)\right) \\
\quad \times \exp \left(\frac{k}{2} \frac{\log ^{2}\left(\left|\tau / q^{\gamma_{3}}\right|\right)}{\log (q)}+\alpha \log \left(\left|\tau / q^{\gamma_{3}}\right|\right)\right) .
\end{aligned}
$$


Hence, using the fact that the contractive map $\tau \mapsto \tau / q^{\gamma_{3}}$ keeps the sector $\bar{S}_{d}$ invariant

$$
\left\|a_{\gamma_{1}}(\tau) \tau^{\gamma_{2}} \sigma_{q, \tau}^{-\gamma_{3}} f(\tau, m)\right\|_{(k, \beta, \mu, \alpha)} \leq \hat{C}_{1}\|f(\tau, m)\|_{(k, \beta, \mu, \alpha)}
$$

with

$$
\begin{aligned}
\hat{C}_{1}= & \sup _{\tau \in \bar{S}_{d}}\left|a_{\gamma_{1}}(\tau)\right||\tau|^{\gamma_{2}} \exp \left(-\frac{k}{2} \frac{\log ^{2}(|\tau|)}{\log (q)}-\alpha \log (|\tau|)\right) \\
& \times \exp \left(\frac{k}{2} \frac{\log ^{2}\left(\left|\tau / q^{\gamma_{3}}\right|\right)}{\log (q)}+\alpha \log \left(\left|\tau / q^{\gamma_{3}}\right|\right)\right) .
\end{aligned}
$$

We can rewrite

$$
\begin{aligned}
\frac{k}{2} \frac{\log ^{2}\left(\left|\tau / q^{\gamma_{3}}\right|\right)}{\log (q)}= & \frac{k}{2 \log (q)}\left(\log ^{2}(|\tau|)-2 \gamma_{3} \log (|\tau|) \log (q)+\left(\gamma_{3} \log (q)\right)^{2}\right), \\
& \alpha \log \left(\left|\tau / q^{\gamma_{3}}\right|\right)=\alpha\left(\log (|\tau|)-\gamma_{3} \log (q)\right)
\end{aligned}
$$

from which we deduce that

$$
\hat{C}_{1} \leq \exp \left(\frac{k}{2} \gamma_{3}^{2} \log (q)-\alpha \gamma_{3} \log (q)\right) \sup _{x \geq 0} \frac{x^{\gamma_{2}-k \gamma_{3}}}{(1+x)^{\gamma_{1}}}
$$

which is finite provided that (4) holds. Gathering the estimates (7), (8), we see that (6) holds.

Definition 2. Let $\beta, \mu \in \boldsymbol{R}$. We denote $E_{(\beta, \mu)}$ the vector space of continuous functions $h: \boldsymbol{R} \rightarrow \boldsymbol{C}$ such that

$$
\|h(m)\|_{(\beta, \mu)}=\sup _{m \in \boldsymbol{R}}(1+|m|)^{\mu} \exp (\beta|m|)|h(m)|
$$

is finite. The space $E_{(\beta, \mu)}$ equipped with the norm $\|\cdot\|_{(\beta, \mu)}$ is a Banach space.

We recall that $\left(E_{(\beta, \mu)},\|\cdot\|_{(\beta, \mu)}\right)$ can be equipped as a Banach algebra for some noncommutative product introduced below (see Proposition 5 of Section 2 in [9]).

Proposition 2. Let $Q(X), R(X) \in C[X]$ be polynomials such that

$$
\operatorname{deg}(R) \geq \operatorname{deg}(Q), \quad R(\text { im }) \neq 0,
$$

for all $m \in \boldsymbol{R}$. Let $m \mapsto b(m)$ be a continuous function on $\boldsymbol{R}$ such that

$$
|b(m)| \leq 1 / \mid R(\text { im }) \mid .
$$


Assume that $\mu>\operatorname{deg}(Q)+1$. Then, there exists a constant $C_{2}>0$ (depending on $Q(X), R(X), \mu)$ such that

$$
\left\|b(m) \int_{-\infty}^{+\infty} f\left(m-m_{1}\right) Q\left(i m_{1}\right) g\left(m_{1}\right) d m_{1}\right\|_{(\beta, \mu)} \leq C_{2}\|f(m)\|_{(\beta, \mu)}\|g(m)\|_{(\beta, \mu)}
$$

for all $f(m), g(m) \in E_{(\beta, \mu)}$. In the sequel we will use the notation

$$
f(m) *^{Q} g(m):=\int_{-\infty}^{+\infty} f\left(m-m_{1}\right) Q\left(\text { im }_{1}\right) g\left(m_{1}\right) d m_{1}
$$

for all $m \in \boldsymbol{R}$, which extends the classical convolution product $*$ in the case $Q \equiv 1$. As a result, $\left(E_{(\beta, \mu)},\|\cdot\|_{(\beta, \mu)}\right)$ becomes a Banach algebra for the product $\star^{b, Q}$ defined by $f(m) \star^{b, Q} g(m):=b(m) f(m) *^{Q} g(m)$.

In the next proposition, we study the continuity property of some $q$-analog of a convolution operator acting on the Banach spaces mentioned above.

Proposition 3. Let $\beta, \mu$ and $b(m), Q(X), R(X)$ chosen as in Proposition 2. Let $c_{h}(m)$ be a sequence of functions belonging to $E_{(\beta, \mu)}$ such that there exist two positive constants $C>0$ and $T>q^{1 /(2 k)} / q^{\alpha / k}$ such that

$$
\left\|c_{h}(m)\right\|_{(\beta, \mu)} \leq C\left(\frac{1}{T}\right)^{h}
$$

for all $h \geq 0$. Let $\varphi_{k}(\tau, m)$ be the power series

$$
\varphi_{k}(\tau, m)=\sum_{h \geq 0} c_{h}(m) \frac{\tau^{h}}{\left(q^{1 / k}\right)^{h(h-1) / 2}}
$$

which defines an entire function w.r.t $\tau$ with values in the Banach space $E_{(\beta, \mu)}$. For any function $f(\tau, m)$ belonging to the Banach space $\operatorname{Exp}_{(k, \beta, \mu, \alpha)}^{q}$, we define a $q$-analog of the convolution of order $k$ of $\varphi_{k}(\tau, m)$ with $f(\tau, m)$ as

$$
\varphi_{k}(\tau, m) * \underset{q ; 1 / k}{Q} f(\tau, m):=\sum_{h \geq 0} \frac{\tau^{h}}{\left(q^{1 / k}\right)^{h(h-1) / 2}} c_{h}(m) * Q\left(\sigma_{q, \tau}^{-h / k} f\right)(\tau, m) .
$$

Then, the function $b(m) \varphi_{k}(\tau, m) *_{q ; 1 / k}^{Q} f(\tau, m)$ belongs to the space $\operatorname{Exp}_{(k, \beta, \mu, \alpha)}^{q}$ and moreover there exists a constant $C_{3}>0$ (depending on $\left.\mu, q, \alpha, k, Q(X), R(X), T\right)$ such that

$$
\left\|b(m) \varphi_{k}(\tau, m) *_{q ; 1 / k}^{Q} f(\tau, m)\right\|_{(k, \beta, \mu, \alpha)} \leq C_{3} C\|f(\tau, m)\|_{(k, \beta, \mu, \alpha)} .
$$


Proof. Let $f(\tau, m) \in \operatorname{Exp}_{(k, \beta, \mu, \alpha)}^{q}$. From the very definition, we know that

$$
\begin{aligned}
& \left\|b(m) \varphi_{k}(\tau, m) *_{q ; 1 / k}^{Q} f(\tau, m)\right\|_{(k, \beta, \mu, \alpha)} \\
& =\sup _{\tau \in \bar{S}_{d}, m \in \boldsymbol{R}}(1+|m|)^{\mu} e^{\beta|m|} \exp \left(-\frac{k}{2} \frac{\log ^{2}(|\tau|)}{\log (q)}-\alpha \log (|\tau|)\right)|b(m)| \\
& \quad \times\left|\sum_{h \geq 0} \frac{\tau^{h}}{\left(q^{1 / k}\right)^{h(h-1) / 2}} \int_{-\infty}^{+\infty} c_{h}\left(m-m_{1}\right) Q\left(i m_{1}\right) f\left(\frac{\tau}{q^{h / k}}, m_{1}\right) d m_{1}\right| .
\end{aligned}
$$

By inserting terms that correspond to the $\|\cdot\|_{(\beta, \mu)}$ norm of $c_{h}(m)$ and to the $\|\cdot\|_{(k, \beta, \mu, \alpha)}$ norm of $f\left(\tau / q^{h / k}, m\right)$, we can give the bound estimates

$$
\begin{aligned}
\left\|b(m) \varphi_{k}(\tau, m) * *_{q ; 1 / k}^{Q} f(\tau, m)\right\|_{(k, \beta, \mu, \alpha)} \\
\leq \sup _{\tau \in \bar{S}_{d}, m \in \boldsymbol{R}}(1+|m|)^{\mu} e^{\beta|m|} \exp \left(-\frac{k}{2} \frac{\log ^{2}(|\tau|)}{\log (q)}-\alpha \log (|\tau|)\right)|b(m)| \\
\times \sum_{h \geq 0} \int_{-\infty}^{+\infty}\left(\left(1+\left|m-m_{1}\right|\right)^{\mu} e^{\beta\left|m-m_{1}\right|} \frac{c_{h}\left(m-m_{1}\right)}{\left.\left(q^{1 / k}\right)^{h(h-1) / 2}|\tau|^{h}\right)}\right. \\
\times\left(\left|f\left(\frac{\tau}{q^{h / k}}, m_{1}\right)\right|\left(1+\left|m_{1}\right|\right)^{\mu} e^{\beta\left|m_{1}\right|}\right. \\
\left.\quad \times \exp \left(-\frac{k}{2} \frac{\log ^{2}\left(\left|\tau / q^{h / k}\right|\right)}{\log (q)}-\alpha \log \left(\left|\tau / q^{h / k}\right|\right)\right)\right) \\
\times\left(\frac{e^{-\beta\left|m-m_{1}\right|}}{\left(1+\left|m-m_{1}\right|\right)^{\mu}} \frac{\left|Q\left(i m_{1}\right)\right|}{\left(1+\left|m_{1}\right|\right)^{\mu}} e^{-\beta\left|m_{1}\right|}\right. \\
\left.\quad \times \exp \left(\frac{k}{2} \frac{\log ^{2}\left(\left|\tau / q^{h / k}\right|\right)}{\log ^{h}(q)}+\alpha \log \left(\left|\tau / q^{h / k}\right|\right)\right)\right) d m_{1} .
\end{aligned}
$$

Using the fact that the contractive map $\tau \mapsto \tau / q^{h / k}$ keeps the sector $\bar{S}_{d}$ invariant, for all $h \geq 0$ and by means of the triangular inequality $|m| \leq\left|m-m_{1}\right|+\left|m_{1}\right|$, we deduce that

$$
\left\|b(m) \varphi_{k}(\tau, m) *_{q ; 1 / k}^{Q} f(\tau, m)\right\|_{(k, \beta, \mu, \alpha)} \leq \hat{C}_{3}\|f(\tau, m)\|_{(k, \beta, \mu, \alpha)}
$$

where 


$$
\begin{aligned}
\hat{C}_{3}= & \sup _{\tau \in \bar{S}_{d}, m \in \boldsymbol{R}}(1+|m|)^{\mu} \exp \left(-\frac{k}{2} \frac{\log ^{2}(|\tau|)}{\log (q)}-\alpha \log (|\tau|)\right)|b(m)| \\
& \times \sum_{h \geq 0}\left\|c_{h}\right\|_{(\beta, \mu)} \frac{|\tau|^{h}}{\left(q^{1 / k}\right)^{h(h-1) / 2}} \int_{-\infty}^{+\infty} \frac{\left|Q\left(i m_{1}\right)\right|}{\left(1+\left|m-m_{1}\right|\right)^{\mu}\left(1+\left|m_{1}\right|\right)^{\mu}} d m_{1} \\
& \times \exp \left(\frac{k}{2} \frac{\log ^{2}\left(\left|\tau / q^{h / k}\right|\right)}{\log (q)}+\alpha \log \left(\left|\tau / q^{h / k}\right|\right)\right)
\end{aligned}
$$

By construction, there exist two constants $\mathfrak{Q}, \mathfrak{R}>0$ such that

$$
\mid Q\left(\text { im }_{1}\right)\left|\leq \mathfrak{Q}\left(1+\left|m_{1}\right|\right)^{\operatorname{deg}(Q)}, \quad\right| R(\text { im }) \mid \geq \mathfrak{R}(1+|m|)^{\operatorname{deg}(R)}
$$

for all $m \in \boldsymbol{R}$. Using (16) and from Lemma 4 in [13] (see also Lemma 2.2 from [3]), we get a constant $\tilde{C}_{3}>0$ with

$$
\begin{aligned}
(1+|m|)^{\mu}|b(m)| \int_{-\infty}^{+\infty} \frac{\left|Q\left(i m_{1}\right)\right|}{\left(1+\left|m-m_{1}\right|\right)^{\mu}\left(1+\left|m_{1}\right|\right)^{\mu}} d m_{1} \\
\leq \sup _{m \in \boldsymbol{R}} \frac{\mathfrak{Q}}{\mathfrak{R}}(1+|m|)^{\mu-\operatorname{deg}(R)} \\
\quad \times \int_{-\infty}^{+\infty} \frac{1}{\left(1+\left|m-m_{1}\right|\right)^{\mu}\left(1+\left|m_{1}\right|\right)^{\mu-\operatorname{deg}(Q)}} d m_{1} \leq \tilde{C}_{3}
\end{aligned}
$$

provided that $\mu>\operatorname{deg}(Q)+1$. On the other hand, we can write

$$
\begin{aligned}
& \frac{k}{2 \log (q)} \log ^{2}\left(\left|\tau / q^{h / k}\right|\right) \\
& \quad=\frac{k}{2 \log (q)}\left(\log ^{2}(|\tau|)-2 \frac{h}{k} \log (|\tau|) \log (q)+\left(\frac{h}{k}\right)^{2} \log ^{2}(q)\right), \\
& \alpha \log \left(\left|\tau / q^{h / k}\right|\right)=\alpha\left(\log (|\tau|)-\frac{h}{k} \log (q)\right) .
\end{aligned}
$$

From (15) and gathering the estimates (11), (17) and the identities (18), we get that

$$
\hat{C}_{3} \leq C \tilde{C}_{3} \sum_{h \geq 0}\left(\frac{q^{1 /(2 k)}}{T q^{\alpha / k}}\right)^{h}
$$

which is finite provided that $T>q^{1 /(2 k)} / q^{\alpha / k}$. Finally, taking into account (14) together with (19) yields the result. 


\subsection{Banach spaces of bounded functions with exponential decay}

We denote $D(0, \rho)$ the open disc centered at 0 with radius $\rho>0$ in $C$ and $\bar{D}(0, \rho)$ its closure.

Definition 3. Let $\beta, \mu, \rho>0$ be real numbers. We denote $\mathcal{B}_{(\beta, \mu, \rho)}$ the vector space of continuous functions $(\tau, m) \mapsto h(\tau, m)$ on $\bar{D}(0, \rho) \times \boldsymbol{R}$, holomorphic w.r.t $\tau$ on $D(0, \rho)$ such that

$$
\|h(\tau, m)\|_{(\beta, \mu, \rho)}=\sup _{\tau \in \bar{D}(0, \rho), m \in \boldsymbol{R}}(1+|m|)^{\mu} e^{\beta|m|}|h(\tau, m)|
$$

is finite. One can check that $\left(\mathcal{B}_{(\beta, \mu, \rho)},\|\cdot\|_{(\beta, \mu, \rho)}\right)$ is a Banach space.

Lemma 2. Let $\gamma \geq 0$ be a real number and $a(\tau, m)$ be a bounded continuous function on $\bar{D}(0, \rho) \times \boldsymbol{R}$, holomorphic on $D(0, \rho)$ w.r.t $\tau$. Then,

$$
\left\|a(\tau, m) \sigma_{q, \tau}^{-\gamma} f(\tau, m)\right\|_{(\beta, \mu, \rho)} \leq \sup _{\tau \in \bar{D}(0, \rho), m \in \boldsymbol{R}}|a(\tau, m)|\|f(\tau, m)\|_{(\beta, \mu, \rho)}
$$

for all $f(\tau, m) \in \mathcal{B}_{(\beta, \mu, \rho)}$.

Proof. Let $f(\tau, m) \in \mathcal{B}_{(\beta, \mu, \rho)}$. Since the transformation $\tau \mapsto \tau / q^{\gamma}$ keeps the disc $\bar{D}(0, \rho)$ invariant, we can write

$$
\begin{aligned}
& \left\|a(\tau, m) \sigma_{q, \tau}^{-\gamma} f(\tau, m)\right\|_{(\beta, \mu, \rho)} \\
& \quad=\sup _{\tau \in \bar{D}(0, \rho), m \in \boldsymbol{R}}(1+|m|)^{\mu} e^{\beta|m|}|a(\tau, m)|\left|f\left(\frac{\tau}{q^{\gamma}}, m\right)\right| \\
& \quad \leq \sup _{\tau \in \bar{D}(0, \rho), m \in \boldsymbol{R}}|a(\tau, m)| \sup _{\tau \in \bar{D}(0, \rho), m \in \boldsymbol{R}}(1+|m|)^{\mu} e^{\beta|m|}|f(\tau, m)|
\end{aligned}
$$

which yields the lemma.

Proposition 4. Let $\beta, \mu$ and $b(m), Q(X), R(X)$ chosen as in Proposition 2. Let $c_{h}(m)$ be a sequence of functions belonging to $E_{(\beta, \mu)}$ such that there exist two positive constants $C, T>0$ such that

$$
\left\|c_{h}(m)\right\|_{(\beta, \mu)} \leq C\left(\frac{1}{T}\right)^{h}
$$

for all $h \geq 0$. Let $\varphi_{k}(\tau, m)$ be the power series

$$
\varphi_{k}(\tau, m)=\sum_{h \geq 0} c_{h}(m) \frac{\tau^{h}}{\left(q^{1 / k}\right)^{h(h-1) / 2}}
$$


which defines an entire function w.r.t $\tau$ with values in the Banach space $E_{(\beta, \mu)}$. For any function $f(\tau, m)$ belonging to the Banach space $\mathcal{B}_{(\beta, \mu, \rho)}$, we define as in Proposition 3 the q-analog of the convolution of order $k$ of $\varphi_{k}(\tau, m)$ with $f(\tau, m)$ as

$$
\varphi_{k}(\tau, m) *{ }_{q ; 1 / k}^{Q} f(\tau, m):=\sum_{h \geq 0} \frac{\tau^{h}}{\left(q^{1 / k}\right)^{h(h-1) / 2}} c_{h}(m) *^{Q}\left(\sigma_{q, \tau}^{-h / k} f\right)(\tau, m) .
$$

Then, $b(m) \varphi_{k}(\tau, m) *_{q ; 1 / k}^{Q} f(\tau, m)$ belongs to the space $\mathcal{B}_{(\beta, \mu, \rho)}$ and moreover there exists a constant $C_{4}>0$ (depending on $\left.\mu, q, \rho, Q(X), R(X), T\right)$ such that

$$
\left\|b(m) \varphi_{k}(\tau, m) *_{q ; 1 / k}^{Q} f(\tau, m)\right\|_{(\beta, \mu, \rho)} \leq C_{4} C\|f(\tau, m)\|_{(\beta, \mu, \rho)} .
$$

Proof. Let $f(\tau, m) \in \mathcal{B}_{(\beta, \mu, \rho)}$. By definition, we have

$$
\begin{aligned}
& \left\|b(m) \varphi_{k}(\tau, m) *_{q ; 1 / k}^{Q} f(\tau, m)\right\|_{(\beta, \mu, \rho)} \\
& \leq \sup _{\tau \in \bar{D}(0, \rho), m \in \boldsymbol{R}}(1+|m|)^{\mu} e^{\beta|m|}|b(m)| \\
& \quad \times\left|\sum_{h \geq 0} \frac{\tau^{h}}{\left(q^{1 / k}\right)^{h(h-1) / 2}} \int_{-\infty}^{+\infty} c_{h}\left(m-m_{1}\right) Q\left(i m_{1}\right) f\left(\frac{\tau}{q^{h / k}}, m_{1}\right) d m_{1}\right| .
\end{aligned}
$$

By enclosing terms related to the $\|\cdot\|_{(\beta, \mu)}$ norm of $c_{h}(m)$ and $\|\cdot\|_{(\beta, \mu, \rho)}$ norm of $f\left(\tau / q^{h / k}, m\right)$, we can provide bounds estimates

$$
\begin{aligned}
& \left\|b(m) \varphi_{k}(\tau, m) *_{q ; 1 / k}^{Q} f(\tau, m)\right\|_{(\beta, \mu, \rho)} \\
& \leq \sup _{\tau \in \bar{D}(0, \rho), m \in \boldsymbol{R}}(1+|m|)^{\mu} e^{\beta|m|}|b(m)| \\
& \quad \times \sum_{h \geq 0} \int_{-\infty}^{+\infty}\left(\left(1+\left|m-m_{1}\right|\right)^{\mu} e^{\beta\left|m-m_{1}\right|}\left|c_{h}\left(m-m_{1}\right)\right| \frac{|\tau|^{h}}{\left(q^{1 / k}\right)^{h(h-1) / 2}}\right) \\
& \quad \times\left(\left|f\left(\frac{\tau}{q^{h / k}}, m_{1}\right)\right|\left(1+\left|m_{1}\right|\right)^{\mu} e^{\beta\left|m_{1}\right|}\right) \\
& \quad \times\left(\frac{e^{-\beta\left|m-m_{1}\right|}}{\left(1+\left|m-m_{1}\right|\right)^{\mu}} \frac{\left|Q\left(i m_{1}\right)\right| e^{-\beta\left|m_{1}\right|}}{\left(1+\left|m_{1}\right|\right)^{\mu}}\right) d m_{1} .
\end{aligned}
$$

Using the fact that the map $\tau \mapsto \tau / q^{h / k}$ keeps the disc $\bar{D}(0, \rho)$ invariant for all $h \geq 0$ and due to the triangular inequality $|m| \leq\left|m-m_{1}\right|+\left|m_{1}\right|$, we get that

$$
\left\|b(m) \varphi_{k}(\tau, m) *_{q ; 1 / k}^{Q} f(\tau, m)\right\|_{(\beta, \mu, \rho)} \leq \hat{C}_{4}\|f(\tau, m)\|_{(\beta, \mu, \rho)},
$$

where 


$$
\begin{aligned}
\hat{C}_{4}= & \sup _{\tau \in \bar{D}(0, \rho), m \in \boldsymbol{R}}(1+|m|)^{\mu}|b(m)| \sum_{h \geq 0}\left\|c_{h}(m)\right\|_{(\beta, \mu)} \frac{|\tau|^{h}}{\left(q^{1 / k}\right)^{h(h-1) / 2}} \\
& \times \int_{-\infty}^{+\infty} \frac{\left|Q\left(i m_{1}\right)\right|}{\left(1+\left|m-m_{1}\right|\right)^{\mu}\left(1+\left|m_{1}\right|\right)^{\mu}} d m_{1} .
\end{aligned}
$$

From the assumption (22) and the estimates (17), we get that

$$
\hat{C}_{4} \leq C \tilde{C}_{3} \sum_{h \geq 0} \frac{1}{\left(q^{1 / k}\right)^{h(h-1) / 2}}\left(\frac{1}{T}\right)^{h} \rho^{h}
$$

which is finite for any $T, \rho>0$. Finally, gathering (26) and (28) yields the result.

In Section 4, we will need the following Banach space:

Definition 4. Let $k, \beta, \mu, \rho>0, q>1$ and $\alpha$ be real numbers. Let $S_{d}$ be an open unbounded sector with bisecting direction $d \in \boldsymbol{R}$ centered at 0 in $\boldsymbol{C}$. We denote $\mathcal{B} \operatorname{Exp}_{(k, \beta, \mu, \alpha, \rho)}^{q}$ the vector space of complex valued continuous functions $(\tau, m) \mapsto h(\tau, m)$ on $\left(\bar{D}(0, \rho) \cup \bar{S}_{d}\right) \times \boldsymbol{R}$, holomorphic w.r.t $\tau$ on $D(0, \rho) \cup S_{d}$ such that

$$
\|h(\tau, m)\|_{(k, \beta, \mu, \alpha, \rho)}=\|h(\tau, m)\|_{(k, \beta, \mu, \alpha)}+\|h(\tau, m)\|_{(\beta, \mu, \rho)}
$$

is finite, where $\|\cdot\|_{(k, \beta, \mu, \alpha)}$ (resp. $\left.\|\cdot\|_{(\beta, \mu, \rho)}\right)$ is the norm defined in Definition 1 (resp. Definition 3). One can check that $\mathcal{B} \operatorname{Exp}_{(k, \beta, \mu, \alpha, \rho)}^{q}$ endowed with the norm $\|\cdot\|_{(k, \beta, \mu, \alpha, \rho)}$ is a Banach space.

\section{3. $q$-Borel, $q$-Laplace and Fourier transforms}

Throughout the whole section we consider a complex Banach space $\boldsymbol{E}$ equipped with the norm $\|\cdot\|_{E}$. Let $q>1$ be a real number and $k \geq 1$ be an integer. We first recall the definition of formal $q$-Borel transform of order $k$ as introduced in [15], Section 4, p. 66.

Definition 5. Let $\hat{a}(T)=\sum_{n \geq 0} a_{n} T^{n} \in \boldsymbol{E}[[T]]$ be a formal series with coefficients $a_{n} \in \boldsymbol{E}$. We define the formal $q$-Borel transform of order $k$ of $\hat{a}(T)$ as the formal series

$$
\hat{\mathcal{B}}_{q ; 1 / k}(\hat{a}(T))(\tau)=\sum_{n \geq 0} a_{n} \frac{\tau^{n}}{\left(q^{1 / k}\right)^{n(n-1) / 2}} \in \boldsymbol{E}[[\tau]] .
$$

When $\gamma \in \boldsymbol{Q}$ is some rational number, the operator $\sigma_{q, T}^{\gamma}$ is acting on $\boldsymbol{E}[[T]]$ as $\left(\sigma_{q, T}^{\gamma} \hat{a}\right)(T):=\hat{a}\left(T q^{\gamma}\right)$ for any formal series $\hat{a}(T) \in \boldsymbol{E}[[T]]$. In the next 
definition, we recall the $q$-analog of the convolution of order $k$ of formal series as set forth in [14], Section 1.4.3 p. 1868.

Definition 6. Assume that the Banach space $\left(\boldsymbol{E},\|\cdot\|_{\boldsymbol{E}}\right)$ has an additional structure of a Banach algebra for some product $\star$ (that may be noncommutative). Let $\hat{A}(\tau)=\sum_{n \geq 0} A_{n} \tau^{n}$ and $\hat{B}(\tau)=\sum_{n \geq 0} B_{n} \tau^{n}$ be two formal power series with coefficients in $\boldsymbol{E}$. We define the $q$-convolution operator of order $k$ of $\hat{B}$ and $\hat{A}$ by the formal series

$$
\hat{B}(\tau) \star_{q ; 1 / k} \hat{A}(\tau)=\sum_{m \geq 0} B_{m} \tau^{m} \star\left(\sigma_{q, \tau}^{-m / k} \hat{A}\right)(\tau) \in \boldsymbol{E}[[\tau]] .
$$

In the next proposition, we recall also some identities for the $q$-Borel transform of order $k$ that will be useful in the sequel. The point 1) may be easily deduced from [6], Lemma 1.2. The point 2) originates from [14], Section 2.4.3 p. 1877.

Proposition 5. 1) Let $\sigma \geq 0$ be an integer and let $j \geq 0$ be a rational number. Then, the identity

$$
\hat{\mathcal{B}}_{q ; 1 / k}\left(T^{\sigma} \sigma_{q, T}^{j} \hat{a}(T)\right)(\tau)=\frac{\tau^{\sigma}}{\left(q^{1 / k}\right)^{\sigma(\sigma-1) / 2}} \sigma_{q, \tau}^{j-\sigma / k}\left(\hat{\mathcal{B}}_{q ; 1 / k}(\hat{a}(T))(\tau)\right)
$$

holds.

2) Let $\hat{a}(T)=\sum_{n \geq 0} a_{n} T^{n}$ and $\hat{b}(T)=\sum_{n \geq 0} b_{n} T^{n}$ be two formal series in $\boldsymbol{E}[[T]]$, where $\boldsymbol{E}$ is equipped with a product $\star$ as in Definition 6. Then, the next identity

$$
\hat{\mathcal{B}}_{q ; 1 / k}(\hat{b}(T) \star \hat{a}(T))(\tau)=\hat{\mathcal{B}}_{q ; 1 / k}(\hat{b}(T))(\tau) \star_{q ; 1 / k} \hat{\mathcal{B}}_{q ; 1 / k}(\hat{b}(T))(\tau)
$$

holds.

Proof. 1) By definition, we can write

$$
\begin{aligned}
\hat{\mathcal{B}}_{q ; 1 / k} & \left(T^{\sigma} \sigma_{q, T}^{j} \hat{a}(T)\right)(\tau) \\
& =\sum_{n \geq 0} \frac{a_{n} q^{j n}}{\left(q^{1 / k}\right)^{(n+\sigma)(n+\sigma-1) / 2}} \tau^{n+\sigma} \\
& =\sum_{n \geq 0} \frac{a_{n}}{\left(q^{1 / k}\right)^{n(n-1) / 2}} \tau^{n}\left(\tau^{\sigma} \frac{q^{j n}}{\left(q^{1 / k}\right)^{(n+\sigma)(n+\sigma-1) / 2}}\left(q^{1 / k}\right)^{n(n-1) / 2}\right) .
\end{aligned}
$$

Since

$$
\frac{q^{j n}}{\left(q^{1 / k}\right)^{(n+\sigma)(n+\sigma-1) / 2}}\left(q^{1 / k}\right)^{n(n-1) / 2}=\frac{1}{\left(q^{1 / k}\right)^{\sigma(\sigma-1) / 2}}\left(q^{j-\sigma / k}\right)^{n}
$$


we deduce from (32) that

$$
\begin{aligned}
\hat{\mathcal{B}}_{q ; 1 / k}\left(T^{\sigma} \sigma_{q, T}^{j} \hat{a}(T)\right)(\tau) & =\frac{\tau^{\sigma}}{\left(q^{1 / k}\right)^{\sigma(\sigma-1) / 2}} \sum_{n \geq 0} \frac{a_{n}}{\left(q^{1 / k}\right)^{n(n-1) / 2}}\left(\tau q^{j-\sigma / k}\right)^{n} \\
& =\frac{\tau^{\sigma}}{\left(q^{1 / k}\right)^{\sigma(\sigma-1) / 2}} \sigma_{q, \tau}^{j-\sigma / k}\left(\hat{\mathcal{B}}_{q ; 1 / k}(\hat{a}(T))(\tau)\right) .
\end{aligned}
$$

2) The second identity (31) is a direct consequence of the formula (30) by taking $j=0$.

We give a definition of a $q$-Laplace transform of order $k$ that extends the $q$-Laplace transform of order 1 which was already used by the author and A. Lastra in [8]. This definition can be seen as a continuous analog of the discrete $q$-Laplace transform of order $k$ used in [6].

Let us first recall some properties of the Jacobi Theta function of order $k$ defined as the Laurent series

$$
\Theta_{q^{1 / k}}(x)=\sum_{n \in \boldsymbol{Z}} q^{-n(n-1) /(2 k)} x^{n}
$$

for all $x \in C^{*}$. This analytic function can be factorized as a product known as the Jacobi's triple product formula,

$$
\Theta_{q^{1 / k}}(x)=\prod_{n \geq 0}\left(1-q^{-(n+1) / k}\right)\left(1+x q^{-n / k}\right)\left(1+\frac{q^{-(n+1) / k}}{x}\right)
$$

for all $x \in C^{*}$, from which we deduce that its zeros is the set of real numbers $\left\{-q^{m / k} \mid m \in \boldsymbol{Z}\right\}$. We remind that $\Theta_{q^{1 / k}}(x)$ solves the $q$-difference equation

$$
\Theta_{q^{1 / k}}\left(q^{m / k} x\right)=q^{m(m+1) /(2 k)} x^{m} \Theta_{q^{1 / k}}(x)
$$

for all $m \in \boldsymbol{Z}$, all $x \in \boldsymbol{C}^{*}$. We recall the next lower bounds estimates on a domain bypassing the set of zeroes of $\Theta_{q^{1 / k}}(x)$, from [8], Lemma 4.1, which are essential in the sequel:

Lemma 3. Let $\delta>0$. There exists a constant $C_{q, k}>0$ depending on $q, k$ and independent of $\delta$ such that

$$
\left|\Theta_{q^{1 / k}}(x)\right| \geq C_{q, k} \delta \exp \left(\frac{k}{2} \frac{\log ^{2}(|x|)}{\log (q)}\right)|x|^{1 / 2}
$$

for all $x \in C^{*}$ satisfying $\left|1+x q^{m / k}\right|>\delta$, for all $m \in \boldsymbol{Z}$.

The following definition of $q$-Laplace transform of order $k$ enhances the one (of order 1) introduced in [22], see also [5], p. 384. 
Definition 7. Let $\rho>0$ be a real number and $S_{d}$ be an unbounded sector centered at 0 with bisecting direction $d \in \boldsymbol{R}$. Let $f: D(0, \rho) \cup S_{d} \rightarrow \boldsymbol{E}$ be a holomorphic function, continuous on $\bar{D}(0, \rho)$, such that there exist constants $K>0$ and $\alpha \in \boldsymbol{R}$ with

$$
\|f(x)\|_{E} \leq K \exp \left(\frac{k}{2} \frac{\log ^{2}(|x|)}{\log (q)}+\alpha \log |x|\right)
$$

for all $x \in S_{d},|x| \geq \rho$ and

$$
\|f(x)\|_{E} \leq K
$$

for all $x \in \bar{D}(0, \rho)$. Let $\gamma \in \boldsymbol{R}$ with $e^{i \gamma} \in S_{d}$. We put $\pi_{q^{1 / k}}=(\log (q) / k) \Pi_{n \geq 0}$. $\left(1-1 / q^{(n+1) / k}\right)^{-1}$. We define the $q$-Laplace transform of order $k$ of $f$ in direction $\gamma$ as

$$
\mathcal{L}_{q ; 1 / k}^{\gamma}(f(x))(T)=\frac{1}{\pi_{q^{1 / k}}} \int_{L_{\gamma}} \frac{f(u)}{\Theta_{q^{1 / k}}(u / T)} \frac{d u}{u}
$$

where $L_{\gamma}=\boldsymbol{R}_{+} e^{i \gamma}$ is a halfline in the direction $\gamma$.

Lemma 4. The integral transform $\mathcal{L}_{q ; 1 / k}^{\gamma}(f(x))(T)$ defines a bounded holomorphic function on the domain $\mathcal{R}_{\gamma, \delta} \cap D\left(0, r_{1}\right)$ for any radius $0<r_{1} \leq$ $q^{(1 / 2-\alpha) / k} / 2$, where

$$
\mathcal{R}_{\gamma, \delta}=\left\{T \in C^{*}|| 1+\frac{e^{i \gamma}}{T} r \mid>\delta, \text { for all } r \geq 0\right\} .
$$

Notice that the value $\mathcal{L}_{q ; 1 / k}^{\gamma}(f(x))(T)$ does not depend on $\gamma \in \boldsymbol{R}$ such that $e^{i \gamma} \in S_{d}$ due to the Cauchy formula.

Proof. From (36), we deduce that

$$
\left\|\int_{L_{\gamma}} \frac{f(u)}{\Theta_{q^{1 / k}}(u / T)} \frac{d u}{u}\right\|_{E} \leq \frac{K}{C_{q, k} \delta}\left(I_{1}(|T|)+I_{2}(|T|)\right)
$$

where

$$
\begin{aligned}
& I_{1}(|T|)=\int_{0}^{\rho} \frac{1}{\exp \left(\frac{k}{2} \frac{\log ^{2}(r /|T|)}{\log (q)}\right)\left(\frac{r}{|T|}\right)^{1 / 2}} \frac{d r}{r}, \\
& I_{2}(|T|)=\int_{\rho}^{+\infty} \frac{\exp \left(\frac{k}{2} \frac{\log ^{2}(r)}{\log (q)}+\alpha \log (r)\right)}{\exp \left(\frac{k}{2} \frac{\log ^{2}(r /|T|)}{\log (q)}\right)\left(\frac{r}{|T|}\right)^{1 / 2}} \frac{d r}{r}
\end{aligned}
$$


for all $T \in \mathcal{R}_{\gamma, \delta} \cap D\left(0, r_{1}\right)$. The first integral $I_{1}(|T|)$ can be rewritten using the change of variable $r^{\prime}=r /|T|$ as

$$
I_{1}(|T|)=\int_{0}^{\rho /|T|} \frac{1}{\exp \left(\frac{k}{2} \frac{\log ^{2}\left(r^{\prime}\right)}{\log (q)}\right)\left(r^{\prime}\right)^{1 / 2}} \frac{d r^{\prime}}{r^{\prime}}
$$

which exists and is bounded w.r.t $|T|$ on $\boldsymbol{R}_{+}$since

$$
\lim _{r^{\prime} \rightarrow 0^{+}} \frac{1}{\exp \left(\frac{k}{2} \frac{\log ^{2}\left(r^{\prime}\right)}{\log (q)}\right) r^{\prime}}=0, \quad \exp \left(\frac{k}{2} \frac{\log ^{2}\left(r^{\prime}\right)}{\log (q)}\right) \geq 1
$$

for all $r^{\prime} \geq 1$. For the second integral, we notice that

$$
\exp \left(\frac{k}{2} \frac{\log ^{2}(r)}{\log (q)}-\frac{k}{2} \frac{\log ^{2}(r /|T|)}{\log (q)}\right)=r^{k \log (|T|) / \log (q)} \exp \left(-\frac{k}{2 \log (q)} \log ^{2}(|T|)\right) .
$$

Therefore, $I_{2}(|T|)$ is bounded w.r.t $|T|$ on $\left[0, q^{(1 / 2-\alpha) / k} / 2\right]$.

In the subsequent proposition, we present some commutation formulas between the $q$-Laplace transform and $q$-difference operator together with multiplication by polynomials.

Proposition 6. Let $f: D(0, \rho) \cup S_{d} \mapsto \boldsymbol{E}$ be a holomorphic function that fulfills the conditions (37), (38). Then, the following formula

$$
T^{\sigma} \sigma_{q, T}^{j}\left(\mathcal{L}_{q ; 1 / k}^{\gamma}(f(x))(T)\right)=\mathcal{L}_{q ; 1 / k}^{\gamma}\left(\frac{x^{\sigma}}{\left(q^{1 / k}\right)^{\sigma(\sigma-1) / 2}} \sigma_{q, x}^{j-\sigma / k} f(x)\right)(T)
$$

holds for all $T \in \mathcal{R}_{\gamma, \delta} \cap D\left(0, r_{1}\right)$ where $0<r_{1} \leq q^{(1 / 2-\alpha) / k} / 2$.

Proof. Using the change of variable $u^{\prime}=q^{j-\sigma / k} u$ in the integral, we can write

$$
\begin{aligned}
& \mathcal{L}_{q ; 1 / k}^{\gamma}\left(\frac{x^{\sigma}}{\left(q^{1 / k}\right)^{\sigma(\sigma-1) / 2}} \sigma_{q, x}^{j-\sigma / k} f(x)\right)(T) \\
& \quad=\frac{1}{\pi_{q^{1 / k}}} \int_{L_{\gamma}} \frac{u^{\sigma} f\left(q^{j-\sigma / k} u\right)}{\left(q^{1 / k}\right)^{\sigma(\sigma-1) / 2} \Theta_{q^{1 / k}}(u / T)} \frac{d u}{u} \\
& \quad=\frac{1}{\pi_{q^{1 / k}}} \int_{L_{\gamma}} \frac{q^{(\sigma / k-j) \sigma} f\left(u^{\prime}\right)\left(u^{\prime}\right)^{\sigma}}{\left(q^{1 / k}\right)^{\sigma(\sigma-1) / 2} \Theta_{q^{1 / k}}\left(q^{\sigma / k-j} u^{\prime} / T\right)} \frac{d u^{\prime}}{u^{\prime}} .
\end{aligned}
$$

On the other hand, from the functional equation (35), the next identity

$$
\frac{q^{(\sigma / k-j) \sigma}\left(u^{\prime}\right)^{\sigma}}{\left(q^{1 / k}\right)^{\sigma(\sigma-1) / 2} \Theta_{q^{1 / k}}\left(q^{\sigma / k}\left(u^{\prime} /\left(q^{j} T\right)\right)\right)}=\frac{T^{\sigma}}{\Theta_{q^{1 / k}}\left(u^{\prime} /\left(q^{j} T\right)\right)}
$$


holds. Gathering (41) and (42) yields that

$$
\begin{aligned}
\mathcal{L}_{q ; 1 / k}^{\gamma}\left(\frac{x^{\sigma}}{\left(q^{1 / k}\right)^{\sigma(\sigma-1) / 2}} \sigma_{q, x}^{j-\sigma / k} f(x)\right)(T) & =\frac{T^{\sigma}}{\pi_{q^{1 / k}}} \int_{L_{\gamma}} \frac{f\left(u^{\prime}\right)}{\Theta_{q^{1 / k}}\left(u^{\prime} /\left(q^{j} T\right)\right)} \frac{d u^{\prime}}{u^{\prime}} \\
& =T^{\sigma} \sigma_{q, T}^{j}\left(\mathcal{L}_{q ; 1 / k}^{\gamma}(f(x))(T)\right) .
\end{aligned}
$$

In the following we recall some well known properties of the Fourier transform already given in [9].

Proposition 7. Let $f \in E_{(\beta, \mu)}$ with $\beta>0, \mu>1$. The inverse Fourier transform of $f$ is defined by

$$
\mathcal{F}^{-1}(f)(x)=\frac{1}{(2 \pi)^{1 / 2}} \int_{-\infty}^{+\infty} f(m) \exp (i x m) d m
$$

for all $x \in \boldsymbol{R}$. The function $\mathcal{F}^{-1}(f)$ extends to an analytic function on the strip

$$
H_{\beta}=\{z \in C|| \operatorname{Im}(z) \mid<\beta\} .
$$

Let $\phi(m)=\operatorname{imf}(m) \in E_{(\beta, \mu-1)}$. Then, we have

$$
\partial_{z} \mathcal{F}^{-1}(f)(z)=\mathcal{F}^{-1}(\phi)(z)
$$

for all $z \in H_{\beta}$.

Let $g \in E_{(\beta, \mu)}$ and let $\psi(m)=(2 \pi)^{-1 / 2} f * g(m)$, the convolution product of $f$ and $g$, for all $m \in \boldsymbol{R}$. From Proposition 2, we know that $\psi \in E_{(\beta, \mu)}$. Moreover, we have

$$
\mathcal{F}^{-1}(f)(z) \mathcal{F}^{-1}(g)(z)=\mathcal{F}^{-1}(\psi)(z)
$$

for all $z \in H_{\beta}$.

\section{Formal and analytic solutions to some auxiliary convolution initial value problems with complex parameters}

Let $k \geq 1$ and $D \geq 3$ be integers. Let $q>1$ be a real number. Let $d_{D} \geq 1$ be an integer. For $1 \leq l \leq D-1$, let $d_{l}, \delta_{l} \geq 1$ and $\Delta_{l} \geq 0$ be nonnegative integers. We make the assumption that

$$
1=\delta_{1}, \quad \delta_{l}<\delta_{l+1},
$$

for all $1 \leq l \leq D-2$. We make also the assumption that

$$
\Delta_{l} \geq d_{l}, \quad \frac{d_{l}}{k}+1 \geq \delta_{l}, \quad \frac{d_{D}}{k}+1>\delta_{l}
$$


for all $1 \leq l \leq D-1$. Let $Q(X), R_{l}(X) \in C[X], 1 \leq l \leq D$, be polynomials such that

$$
\operatorname{deg}(Q) \geq \operatorname{deg}\left(R_{D}\right) \geq \operatorname{deg}\left(R_{l}\right), \quad Q(\text { im }) \neq 0, \quad R_{D}(\text { im }) \neq 0
$$

for all $m \in \boldsymbol{R}$, all $1 \leq l \leq D-1$. We consider sequences of functions $m \mapsto$ $C_{l, n}(m, \varepsilon)$ and $m \mapsto F_{n}(m, \varepsilon)$ for all $n \geq 0,1 \leq l \leq D-1$ that belong to the Banach space $E_{(\beta, \mu)}$ for some $\beta>0$ and $\mu>\operatorname{deg}\left(R_{D}\right)+1$ and which depend holomorphically on $\varepsilon \in D\left(0, \varepsilon_{0}\right)$ for some $\varepsilon_{0}>0$. We assume that there exist constants $\tilde{C}_{l}, C_{F}, T_{0}>0$ such that

$$
\left\|C_{l, n}(m, \varepsilon)\right\|_{(\beta, \mu)} \leq \tilde{C}_{l}\left(\frac{1}{T_{0}}\right)^{n}, \quad\left\|F_{n}(m, \varepsilon)\right\|_{(\beta, \mu)} \leq C_{F}\left(\frac{1}{T_{0}}\right)^{n}
$$

for all $1 \leq l \leq D-1$, for all $n \geq 0$, for all $\varepsilon \in D\left(0, \varepsilon_{0}\right)$. We define

$$
C_{l}(T, m, \varepsilon)=\sum_{n \geq 0} C_{l, n}(m, \varepsilon) T^{n}, \quad F(T, m, \varepsilon)=\sum_{n \geq 0} F_{n}(m, \varepsilon) T^{n}
$$

which are convergent series on $D\left(0, T_{0} / 2\right)$ with values in $E_{(\beta, \mu)}$. We consider the following initial value problem

$$
\begin{aligned}
Q(\text { im }) & \sigma_{q, T} U(T, m, \varepsilon) \\
= & T^{d_{D}} \sigma_{q, T}^{d_{D} / k+1} R_{D}(i m) U(T, m, \varepsilon)+\sum_{l=1}^{D-1} \varepsilon^{A_{l}-d_{l}} T^{d_{l}} \sigma_{q, T}^{\delta_{l}} \\
& \times\left(\frac{1}{(2 \pi)^{1 / 2}} \int_{-\infty}^{+\infty} C_{l}\left(T, m-m_{1}, \varepsilon\right) R_{l}\left(i m_{1}\right) U\left(T, m_{1}, \varepsilon\right) d m_{1}\right) \\
& +\sigma_{q, T} F(T, m, \varepsilon) .
\end{aligned}
$$

Proposition 8. There exists a unique formal power series

$$
\hat{U}(T, m, \varepsilon)=\sum_{n \geq 0} U_{n}(m, \varepsilon) T^{n}
$$

solution of (50), where the coefficients $U_{n}(m, \varepsilon)$ belong to $E_{(\beta, \mu)}$, for $\beta>0$ and $\mu>\operatorname{deg}\left(R_{D}\right)+1$ given above and depend holomorphically on $\varepsilon \in D\left(0, \varepsilon_{0}\right)$.

Proof. From Proposition 2 and the conditions (48), we deduce that all the coefficients $U_{n}(m, \varepsilon)$ are well defined and belong to $E_{(\beta, \mu)}$, for all $\varepsilon \in D\left(0, \varepsilon_{0}\right)$ since they satisfy the next recursion relation 
(51)

$$
\begin{aligned}
Q(\text { im }) & U_{n}(m, \varepsilon) q^{n} \\
= & R_{D}(\text { im }) U_{n-d_{D}}(m, \varepsilon) q^{\left(d_{D} / k+1\right)\left(n-d_{D}\right)}+\sum_{l=1}^{D-1} \varepsilon^{\Delta_{l}-d_{l}} q^{\left(n-d_{l}\right) \delta_{l}} \\
& \times\left(\sum_{n_{1}+n_{2}=n-d_{l}} \frac{1}{(2 \pi)^{1 / 2}} \int_{-\infty}^{+\infty} C_{l, n_{1}}\left(m-m_{1}, \varepsilon\right) R_{l}\left(i m_{1}\right) U_{n_{2}}\left(m_{1}, \varepsilon\right) d m_{1}\right) \\
& +F_{n}(m, \varepsilon) q^{n}
\end{aligned}
$$

for all $n \geq \max _{1 \leq l \leq D} d_{l}$.

In the next step, we rewrite the equation (50) in multiplying its left and right handside by $T^{k}$ in order to get corresponding equations for the formal $q$-Borel transform of $\hat{U}(T, m, \varepsilon)$ w.r.t $T$, namely

$$
\begin{aligned}
Q(i m) & T^{k} \sigma_{q, T} U(T, m, \varepsilon) \\
= & T^{d_{D}+k} \sigma_{q, T}^{d_{D} / k+1} R_{D}(i m) U(T, m, \varepsilon)+\sum_{l=1}^{D-1} \varepsilon^{\Delta_{l}-d_{l}} T^{d_{l}+k} \sigma_{q, T}^{\delta_{l}} \\
& \times\left(\frac{1}{(2 \pi)^{1 / 2}} \int_{-\infty}^{+\infty} C_{l}\left(T, m-m_{1}, \varepsilon\right) R_{l}\left(i m_{1}\right) U\left(T, m_{1}, \varepsilon\right) d m_{1}\right) \\
& +T^{k} \sigma_{q, T} F(T, m, \varepsilon) .
\end{aligned}
$$

We denote $\omega_{k}(\tau, m, \varepsilon)$ the formal $q$-Borel transform of order $k$ of $\hat{U}(T, m, \varepsilon)$ with respect to $T, \varphi_{k, l}(\tau, m, \varepsilon)$ the formal $q$-Borel transform of order $k$ of $C_{l}(T, m, \varepsilon)$ with respect to $T$ and $\psi_{k}(\tau, m, \varepsilon)$ the formal $q$-Borel transform of order $k$ of $F(T, m, \varepsilon)$ with respect to $T$, written as

$$
\begin{aligned}
& \omega_{k}(\tau, m, \varepsilon)=\sum_{n \geq 0} U_{n}(m, \varepsilon) \frac{\tau^{n}}{\left(q^{1 / k}\right)^{n(n-1) / 2}}, \\
& \varphi_{k, l}(\tau, m, \varepsilon)=\sum_{n \geq 0} C_{l, n}(m, \varepsilon) \frac{\tau^{n}}{\left(q^{1 / k}\right)^{n(n-1) / 2}}, \\
& \psi_{k}(\tau, m, \varepsilon)=\sum_{n \geq 0} F_{n}(m, \varepsilon) \frac{\tau^{n}}{\left(q^{1 / k}\right)^{n(n-1) / 2}} .
\end{aligned}
$$

Lemma 5. Let $T_{0}>0$ be fixed as in (49). We take a real number $\alpha \in \boldsymbol{R}$ such that

$$
T_{0}>q^{1 /(2 k)} / q^{\alpha / k}
$$


Let $k, \beta, \mu$ be chosen as above. Then, the function $\psi_{k}(\tau, m, \varepsilon)$ belongs to the Banach space $\mathcal{B} \operatorname{Exp}_{(k, \beta, \mu, \alpha, \rho)}^{q}$ for any unbounded sector $S_{d}$ and any disc $D(0, \rho)$.

Proof. Using (49), we get

$$
\begin{aligned}
& \left\|\psi_{k}(\tau, m, \varepsilon)\right\|_{(k, \beta, \mu, \alpha)} \\
& \quad \leq \sum_{n \geq 0}\left\|F_{n}(m, \varepsilon) \frac{\tau^{n}}{\left(q^{1 / k}\right)^{n(n-1) / 2}}\right\|_{(k, \beta, \mu, \alpha)} \\
& \quad \leq \sum_{n \geq 0} C_{F}\left(\frac{1}{T_{0}}\right)^{n}\left(\sup _{\tau \in \bar{S}_{d}}|\tau|^{n} \exp \left(-\frac{k}{2} \frac{\log ^{2}|\tau|}{\log (q)}-\alpha \log |\tau|\right)\right) \frac{1}{\left(q^{1 / k}\right)^{n(n-1) / 2}}
\end{aligned}
$$

Now, we observe that

$$
\begin{aligned}
& \sup _{\tau \in \bar{S}_{d}}|\tau|^{n} \exp \left(-\frac{k}{2} \frac{\log ^{2}|\tau|}{\log (q)}-\alpha \log |\tau|\right) \\
& \quad=\sup _{x \in \boldsymbol{R}} \exp \left(x(n-\alpha)-\frac{k}{2} \frac{x^{2}}{\log (q)}\right) \leq q^{(n-\alpha)^{2} /(2 k)}
\end{aligned}
$$

by using the change of variable $x=\log |\tau|$ and the fact that the function $h(x)=x(n-\alpha)-(k / 2) x^{2} / \log (q)$ gets its maximum value $(\log (q) /(2 k))(n-\alpha)^{2}$ at $x=(\log (q) / k)(n-\alpha)$. From (54) and (55), we deduce that

$$
\left\|\psi_{k}(\tau, m, \varepsilon)\right\|_{(k, \beta, \mu, \alpha)} \leq C_{F} q^{\alpha^{2} /(2 k)} \sum_{n \geq 0}\left(\frac{q^{1 /(2 k)}}{T_{0} q^{\alpha / k}}\right)^{n}
$$

which converges provided that $T_{0}>q^{1 /(2 k)} / q^{\alpha / k}$. In the second part of the proof, we show that $\psi_{k}(\tau, m, \varepsilon)$ also belongs to the space $\mathcal{B}_{(\beta, \mu, \rho)}$. Namely, taking again (49) into account yields

$$
\begin{aligned}
\left\|\psi_{k}(\tau, m, \varepsilon)\right\|_{(\beta, \mu, \rho)} & \leq \sum_{n \geq 0}\left\|F_{n}(m, \varepsilon) \frac{\tau^{n}}{\left(q^{1 / k}\right)^{n(n-1) / 2}}\right\|_{(\beta, \mu, \rho)} \\
& \leq C_{F} \sum_{n \geq 0} \frac{\left(\rho / T_{0}\right)^{n}}{\left(q^{1 / k}\right)^{n(n-1) / 2}}
\end{aligned}
$$

that converges for any given $\rho>0$. In consequence, we see that $\psi_{k}(\tau, m, \varepsilon)$ belongs to the space $\mathcal{B} \operatorname{Exp}_{(k, \beta, \mu, \alpha, \rho)}^{q}$.

Applying the commutation rules (30) and (31) in Proposition 5 in the framework of the Banach algebra products $\star^{b, Q}$ introduced in Proposition 2, applied to (52) yields the following equation for $\omega_{k}(\tau, m, \varepsilon)$, 


$$
\begin{aligned}
Q(i m) & \frac{\tau^{k}}{\left(q^{1 / k}\right)^{k(k-1) / 2}} \omega_{k}(\tau, m, \varepsilon) \\
= & R_{D}(i m) \frac{\tau^{d_{D}+k}}{\left(q^{1 / k}\right)^{\left(d_{D}+k\right)\left(d_{D}+k-1\right) / 2}} \omega_{k}(\tau, m, \varepsilon) \\
& +\sum_{l=1}^{D-1} \varepsilon^{\Delta_{l}-d_{l}} \frac{\tau^{d_{l}+k}}{\left(q^{1 / k}\right)^{\left(d_{l}+k\right)\left(d_{l}+k-1\right) / 2}} \sigma_{q, \tau}^{\delta_{l}-d_{l} / k-1} \\
& \times\left(\frac{1}{(2 \pi)^{1 / 2}} \varphi_{k, l}(\tau, m, \varepsilon) *_{q ; 1 / k}^{R_{l}} \omega_{k}(\tau, m, \varepsilon)\right) \\
& +\frac{\tau^{k}}{\left(q^{1 / k}\right)^{k(k-1) / 2}} \psi_{k}(\tau, m, \varepsilon) .
\end{aligned}
$$

We make the further assumption that there exists an unbounded sector

$$
S_{Q, R_{D}}=\left\{z \in C|| z\left|\geq r_{Q, R_{D}},\right| \arg (z)-d_{Q, R_{D}} \mid \leq \eta_{Q, R_{D}}\right\}
$$

with direction $d_{Q, R_{D}} \in \boldsymbol{R}$, aperture $\eta_{Q, R_{D}}>0$ for some radius $r_{Q, R_{D}}>0$ such that

$$
\frac{Q(i m)}{R_{D}(i m)} \in S_{Q, R_{D}}
$$

for all $m \in \boldsymbol{R}$. We factorize the polynomial

$$
P_{m}(\tau)=\frac{Q(i m)}{\left(q^{1 / k}\right)^{k(k-1) / 2}}-\frac{R_{D}(i m)}{\left(q^{1 / k}\right)^{\left(d_{D}+k\right)\left(d_{D}+k-1\right) / 2}} \tau^{d_{D}}
$$

in the form

$$
P_{m}(\tau)=-\frac{R_{D}(i m)}{\left(q^{1 / k}\right)^{\left(d_{D}+k\right)\left(d_{D}+k-1\right) / 2}} \Pi_{l=0}^{d_{D}-1}\left(\tau-q_{l}(m)\right)
$$

where

$$
\text { (62) } \begin{aligned}
q_{l}(m)= & \left(\frac{|Q(i m)|}{\left|R_{D}(i m)\right|}\left(q^{1 / k}\right)^{\left\{\left(d_{D}+k\right)\left(d_{D}+k-1\right)-k(k-1)\right\} / 2}\right)^{1 / d_{D}} \\
& \times \exp \left(\sqrt{-1}\left(\frac{1}{d_{D}} \arg \left(\frac{Q(i m)}{R_{D}(\text { im })}\left(q^{1 / k}\right)^{\left\{\left(d_{D}+k\right)\left(d_{D}+k-1\right)-k(k-1)\right\} / 2}\right)+\frac{2 \pi l}{d_{D}}\right)\right)
\end{aligned}
$$

for all $0 \leq l \leq d_{D}-1$. We choose an unbounded sector $S_{d}$ centered at 0 , a small closed disc $\bar{D}(0, \rho)$ and we prescribe the sector $S_{Q, R_{D}}$ in such a way that the following conditions hold. 
1) There exists a constant $M_{1}>0$ such that

$$
\left|\tau-q_{l}(m)\right| \geq M_{1}(1+|\tau|)
$$

for all $0 \leq l \leq d_{D}-1$, all $m \in \boldsymbol{R}$, all $\tau \in S_{d} \cup \bar{D}(0, \rho)$. Indeed, from (59) and the explicit expression (62) of $q_{l}(m)$, we first observe that $\left|q_{l}(m)\right|>2 \rho$ for every $m \in \boldsymbol{R}$, all $0 \leq l \leq d_{D}-1$ for an appropriate choice of $r_{Q, R_{D}}$ and of $\rho>0$. We also see that for all $m \in \boldsymbol{R}$, all $0 \leq l \leq d_{D}-1$, the roots $q_{l}(m)$ remain in a union $\mathcal{U}$ of unbounded sectors centered at 0 that do not cover a full neighborhood of the origin in $C^{*}$ provided that $\eta_{Q, R_{D}}$ is small enough. Therefore, one can choose an adequate sector $S_{d}$ such that $S_{d} \cap \mathcal{U}=\varnothing$ with the property that for all $0 \leq l \leq d_{D}-1$ the quotients $q_{l}(m) / \tau$ lay outside some small disc centered at 1 in $\boldsymbol{C}$ for all $\tau \in S_{d}$, all $m \in \boldsymbol{R}$. This yields (63) for some small constant $M_{1}>0$.

2) There exists a constant $M_{2}>0$ such that

$$
\left|\tau-q_{l_{0}}(m)\right| \geq M_{2}\left|q_{l_{0}}(m)\right|
$$

for some $l_{0} \in\left\{0, \ldots, d_{D}-1\right\}$, all $m \in \boldsymbol{R}$, all $\tau \in S_{d} \cup \bar{D}(0, \rho)$. Indeed, for the sector $S_{d}$ and the disc $\bar{D}(0, \rho)$ chosen as above in 1), we notice that for any fixed $0 \leq l_{0} \leq d_{D}-1$, the quotient $\tau / q_{l_{0}}(m)$ stays outside a small disc centered at 1 in $\boldsymbol{C}$ for all $\tau \in S_{d} \cup \bar{D}(0, \rho)$, all $m \in \boldsymbol{R}$. Hence (64) must hold for some small constant $M_{2}>0$.

By construction of the roots (62) in the factorization (61) and using the lower bound estimates (63), (64), we get a constant $C_{P}>0$ such that

$$
\begin{aligned}
\left|P_{m}(\tau)\right| \geq & M_{1}^{d_{D}-1} M_{2} \frac{\mid R_{D}(\text { im }) \mid}{\left(q^{1 / k}\right)^{\left(d_{D}+k\right)\left(d_{D}+k-1\right) / 2}} \\
& \times\left(\frac{|Q(i m)|}{\left|R_{D}(i m)\right|}\left(q^{1 / k}\right)^{\left\{\left(d_{D}+k\right)\left(d_{D}+k-1\right)-k(k-1)\right\} / 2}\right)^{1 / d_{D}} \\
& \times(1+|\tau|)^{d_{D}-1} \geq C_{P}\left(r_{Q, R_{D}}\right)^{1 / d_{D}} \mid R_{D}(\text { im }) \mid(1+|\tau|)^{d_{D}-1}
\end{aligned}
$$

for all $\tau \in S_{d} \cup \bar{D}(0, \rho)$, all $m \in \boldsymbol{R}$.

In the next proposition, we present sufficient conditions under which the equation (58) has a unique solution in the Banach space $\mathcal{B} \operatorname{Exp}_{(k, \beta, \mu, \alpha, \rho)}^{q}$ where $k, \beta, \mu, \alpha, \rho$ and the sector $S_{d}$ are prescribed as above.

Proposition 9. Under the assumptions (46) and (47) there exist a radius $r_{Q, R_{D}}>0$, a constant $\varpi>0$ and constants $\zeta_{l}, \zeta_{\psi}>0$ such that if

$$
\tilde{C}_{l} \leq \zeta_{l}, \quad C_{F} \leq \zeta_{\psi}
$$

for all $1 \leq l \leq D-1$, where $\tilde{C}_{l}$ are defined in (49), then the equation (58) has a unique solution $\omega_{k}^{d}(\tau, m, \varepsilon)$ in the space $\mathcal{B} \operatorname{Exp}_{(k, \beta, \mu, \alpha, \rho)}^{q}$ which satisfies $\left\|\omega_{k}^{d}(\tau, m, \varepsilon)\right\|_{(k, \beta, \mu, \alpha, \rho)} \leq \varpi$, for all $\varepsilon \in D\left(0, \varepsilon_{0}\right)$. 
Proof. We start the proof with a lemma.

Lemma 6. One can choose a constant $r_{Q, R_{D}}>0$ large enough, a constant $\varpi>0$ small enough and constants $\zeta_{l}, \zeta_{\psi}>0$ submitted to (66) for $1 \leq l \leq D-1$, the map $\mathcal{H}_{\varepsilon}$ defined as

$$
\begin{aligned}
\mathcal{H}_{\varepsilon}(w(\tau, m)):= & \sum_{l=1}^{D-1} \varepsilon^{\Delta_{l}-d_{l}} \frac{\tau^{d_{l}}}{P_{m}(\tau)\left(q^{1 / k}\right)^{\left(d_{l}+k\right)\left(d_{l}+k-1\right) / 2}} \\
& \times \sigma_{q, \tau}^{\delta_{l}-d_{l} / k-1}\left(\frac{1}{(2 \pi)^{1 / 2}} \varphi_{k, l}(\tau, m, \varepsilon) *_{q ; 1 / k}^{R_{l}} w(\tau, m)\right) \\
& +\frac{1}{P_{m}(\tau)\left(q^{1 / k}\right)^{k(k-1) / 2}} \psi_{k}(\tau, m, \varepsilon)
\end{aligned}
$$

satisfies the next two properties:

i) The following inclusion

$$
\mathcal{H}_{\varepsilon}(\bar{B}(0, \varpi)) \subset \bar{B}(0, \varpi)
$$

holds, where $\bar{B}(0, \varpi)$ denotes the closed ball of radius $\varpi>0$ centered at 0 in $\mathcal{B} \operatorname{Exp}_{(k, \beta, \mu, \alpha, \rho)}^{q}$, for all $\varepsilon \in D\left(0, \varepsilon_{0}\right)$.

ii) The inequality

(69) $\left\|\mathscr{H}_{\varepsilon}\left(w_{1}(\tau, m)\right)-\mathscr{H}_{\varepsilon}\left(w_{2}(\tau, m)\right)\right\|_{(k, \beta, \mu, \alpha, \rho)} \leq \frac{1}{2}\left\|w_{1}(\tau, m)-w_{2}(\tau, m)\right\|_{(k, \beta, \mu, \alpha, \rho)}$

holds for all $w_{1}(\tau, m), w_{2}(\tau, m) \in \bar{B}(0, \varpi)$, for all $\varepsilon \in D\left(0, \varepsilon_{0}\right)$.

Proof. We first check i). Let $w(\tau, m) \in \mathcal{B} \operatorname{Exp}_{(k, \beta, \mu, \alpha, \rho)}^{q}$. We choose $\varpi, \zeta_{l}, \zeta_{\psi}>0, \quad 1 \leq l \leq D-1$ such that $\|w(\tau, m)\|_{(k, \beta, \mu, \alpha, \rho)} \leq \varpi$ and (66) hold for all $\varepsilon \in D\left(0, \varepsilon_{0}\right)$.

Bearing in mind the assumptions (46), (47), (48), (49) under the restriction (53) together with the lower bounds (65), we make use of Proposition 1 and Proposition 3 in order to get the estimates

$$
\begin{aligned}
& \| \varepsilon^{\Delta_{l}-d_{l}} \frac{\tau^{d_{l}}}{P_{m}(\tau)\left(q^{1 / k}\right)^{\left(d_{l}+k\right)\left(d_{l}+k-1\right) / 2}} \\
& \quad \times \sigma_{q, \tau}^{\delta_{l}-d_{l} / k-1}\left(\frac{1}{(2 \pi)^{1 / 2}} \varphi_{k, l}(\tau, m, \varepsilon) *_{q ; 1 / k}^{R_{l}} w(\tau, m)\right) \|_{(k, \beta, \mu, \alpha)} \\
& \leq \varepsilon_{0}^{\Delta_{l}-d_{l}} \tilde{C}_{l} \frac{C_{1} C_{3}}{\left(q^{1 / k}\right)^{\left(d_{l}+k\right)\left(d_{l}+k-1\right) / 2} C_{P}\left(r_{Q, R_{D}}\right)^{1 / d_{D}}(2 \pi)^{1 / 2}}\|w(\tau, m)\|_{(k, \beta, \mu, \alpha)},
\end{aligned}
$$


moreover, from Lemma 2 and Proposition 4, we also get

$$
\begin{aligned}
\| \varepsilon^{\Delta_{l}-d_{l}} & \frac{\tau^{d_{l}}}{P_{m}(\tau)\left(q^{1 / k}\right)^{\left(d_{l}+k\right)\left(d_{l}+k-1\right) / 2}} \\
& \times \sigma_{q, \tau}^{\delta_{l}-d_{l} / k-1}\left(\frac{1}{(2 \pi)^{1 / 2}} \varphi_{k, l}(\tau, m, \varepsilon) *_{q ; 1 / k}^{R_{l}} w(\tau, m)\right) \|_{(\beta, \mu, \rho)} \\
\leq & \varepsilon_{0}^{\Delta_{l}-d_{l}} \tilde{C}_{l} \frac{\rho^{d_{l}} C_{4}}{\left(q^{1 / k}\right)^{\left(d_{l}+k\right)\left(d_{l}+k-1\right) / 2} C_{P}\left(r_{Q, R_{D}}\right)^{1 / d_{D}}(2 \pi)^{1 / 2}}\|w(\tau, m)\|_{(\beta, \mu, \rho)} .
\end{aligned}
$$

Gathering (70) and (71) yields the norm estimates

$$
\begin{aligned}
\| \varepsilon^{\Delta_{l}-d_{l}} & \frac{\tau^{d_{l}}}{P_{m}(\tau)\left(q^{1 / k}\right)^{\left(d_{l}+k\right)\left(d_{l}+k-1\right) / 2}} \\
& \times \sigma_{q, \tau}^{\delta_{l}-d_{l} / k-1}\left(\frac{1}{(2 \pi)^{1 / 2}} \varphi_{k, l}(\tau, m, \varepsilon) *_{q ; 1 / k}^{R_{l}} w(\tau, m)\right) \\
\leq & \varepsilon_{0}^{\Delta_{l}-d_{l}} \zeta_{l} \frac{\max \left(C_{1} C_{3}, \rho^{d_{l}} C_{4}\right)}{\left(q^{1 / k}\right)^{\left(d_{l}+k\right)\left(d_{l}+k-1\right) / 2} C_{P}\left(r_{Q, R_{D}}\right)^{1 / d_{D}}(2 \pi)^{1 / 2}} \varpi
\end{aligned}
$$

for all $1 \leq l \leq D-1$. On the other hand, from Lemma 1 and Lemma 2, and bearing in mind the estimates (56) and (57) we deduce that

$$
\begin{aligned}
& \left\|\frac{1}{P_{m}(\tau)\left(q^{1 / k}\right)^{k(k-1) / 2}} \psi_{k}(\tau, m, \varepsilon)\right\|_{(k, \beta, \mu, \alpha, \rho)} \\
& \leq \frac{1}{\left(q^{1 / k}\right)^{k(k-1) / 2} C_{P}\left(r_{Q, R_{D}}\right)^{1 / d_{D}}} \sup _{m \in \boldsymbol{R}} \frac{1}{\left|R_{D}(i m)\right|} \zeta_{\psi} \\
& \quad \times\left(q^{\alpha^{2} /(2 k)} \sum_{n \geq 0}\left(\frac{q^{1 /(2 k)}}{T_{0} q^{\alpha / k}}\right)^{n}+\sum_{n \geq 0} \frac{\left(\rho / T_{0}\right)^{n}}{\left(q^{1 / k}\right)^{n(n-1) / 2}}\right) .
\end{aligned}
$$

Now, we choose $r_{Q, R_{D}}>0$ and $\varpi, \zeta_{l}, \zeta_{\psi}>0,1 \leq l \leq D-1$ in order that

$$
\begin{aligned}
& \sum_{l=1}^{D-1} \varepsilon_{0}^{\Delta_{l}-d_{l}} \zeta_{l} \frac{\max \left(C_{1} C_{3}, \rho^{d_{l}} C_{4}\right)}{\left(q^{1 / k}\right)^{\left(d_{l}+k\right)\left(d_{l}+k-1\right) / 2} C_{P}\left(r_{Q, R_{D}}\right)^{1 / d_{D}}(2 \pi)^{1 / 2}} \varpi \\
& \quad+\frac{1}{\left(q^{1 / k}\right)^{k(k-1) / 2} C_{P}\left(r_{Q, R_{D}}\right)^{1 / d_{D}}} \sup _{m \in \boldsymbol{R}} \frac{1}{\left|R_{D}(i m)\right|} \zeta_{\psi} \\
& \quad \times\left(q^{\alpha^{2} /(2 k)} \sum_{n \geq 0}\left(\frac{q^{1 /(2 k)}}{T_{0} q^{\alpha / k}}\right)^{n}+\sum_{n \geq 0} \frac{\left(\rho / T_{0}\right)^{n}}{\left(q^{1 / k}\right)^{n(n-1) / 2}}\right) \leq \varpi .
\end{aligned}
$$


Taking into account the above estimates (72) and (73) under the constraint (74), one obtains (68).

In a second step, we check ii). Let $w_{1}, w_{2} \in \mathcal{B} \operatorname{Exp}_{(k, \beta, \mu, \alpha, \rho)}^{q}$. Let $\varpi>0$ such that $\left\|w_{l}(\tau, m)\right\|_{(k, \beta, \mu, \alpha, \rho)} \leq \varpi, l=1,2$. From the estimates (72) we see at once that

$$
\begin{aligned}
& \| \varepsilon^{\Delta_{l}-d_{l}} \frac{\tau^{d_{l}}}{P_{m}(\tau)\left(q^{1 / k}\right)^{\left(d_{l}+k\right)\left(d_{l}+k-1\right) / 2}} \\
& \quad \times \sigma_{q, \tau}^{\delta_{l}-d_{l} / k-1}\left(\frac{1}{(2 \pi)^{1 / 2}} \varphi_{k, l}(\tau, m, \varepsilon) *_{q ; 1 / k}^{R_{l}}\left(w_{1}(\tau, m)-w_{2}(\tau, m)\right)\right) \|_{(k, \beta, \mu, \alpha, \rho)} \\
& \leq \varepsilon_{0}^{\Delta_{l}-d_{l}} \zeta_{l} \frac{\max \left(C_{1} C_{3}, \rho^{d_{l}} C_{4}\right)}{\left(q^{1 / k}\right)^{\left(d_{l}+k\right)\left(d_{l}+k-1\right) / 2} C_{P}\left(r_{Q, R_{D}}\right)^{1 / d_{D}}(2 \pi)^{1 / 2}} \\
& \quad \times\left\|w_{1}(\tau, m)-w_{2}(\tau, m)\right\|_{(k, \beta, \mu, \alpha, \rho)} .
\end{aligned}
$$

Now we select $r_{Q, R_{D}}>0$ and $\zeta_{l}>0,1 \leq l \leq D-1$, such that

$$
\sum_{l=1}^{D-1} \varepsilon_{0}^{\Delta_{l}-d_{l}} \zeta_{l} \frac{\max \left(C_{1} C_{3}, \rho^{d_{l}} C_{4}\right)}{\left(q^{1 / k}\right)^{\left(d_{l}+k\right)\left(d_{l}+k-1\right) / 2} C_{P}\left(r_{Q, R_{D}}\right)^{1 / d_{D}}(2 \pi)^{1 / 2}} \leq \frac{1}{2}
$$

Hence due to (75) under the constraint (76), we get that (69).

Finally, we single out constants $\varpi, \zeta_{l}, \zeta_{\psi}>0$ and $r_{Q, R_{D}}>0$ in such a way that both (74) and (76) hold. This provides our lemma.

We consider the ball $\bar{B}(0, \varpi) \subset \mathcal{B} \operatorname{Exp}_{(k, \beta, \mu, \alpha, \rho)}^{q}$ determined in the lemma above, which defines a complete metric space for the norm $\|\cdot\|_{(k, \beta, \mu, \alpha, \rho)}$. From the lemma 6 , we get that $\mathcal{H}_{\varepsilon}$ is a contractive map from $\bar{B}(0, \varpi)$ into itself. Due to the classical contractive mapping theorem we deduce that $\mathcal{H}_{\varepsilon}$ has a unique fixed point denoted $\omega_{k}^{d}(\tau, m, \varepsilon)$ for all $\varepsilon \in D\left(0, \varepsilon_{0}\right)$. Moreover, $\omega_{k}^{d}(\tau, m, \varepsilon)$ depends holomorphically on $\varepsilon$ on $D\left(0, \varepsilon_{0}\right)$. By construction, $\omega_{k}^{d}$ solves the problem (58).

In the next proposition, we provide analytic solutions to our main convolution equation (50).

Proposition 10. Under the assumptions (46), (47), (48) and (49) together with (53), provided that the sector $S_{d}$ and the disc $D(0, \rho)$ fulfill the constraints (63), (64) and under the restriction of constants (66), the equation (50) gets a solution $(T, m, \varepsilon) \mapsto U^{d}(T, m, \varepsilon)$ that can be expressed as a q-Laplace transform of order $k$ in direction $d$,

$$
U^{d}(T, m, \varepsilon)=\frac{1}{\pi_{q^{1 / k}}} \int_{L_{d}} \frac{\omega_{k}^{d}(u, m, \varepsilon)}{\Theta_{q^{1 / k}}(u / T)} \frac{d u}{u},
$$


where $\omega_{k}^{d}(u, m, \varepsilon)$ belongs to the Banach space $\mathcal{B} \operatorname{Exp}_{(k, \beta, \mu, \alpha, \rho)}^{q}$ for the sector $S_{d}$ and the disc $D(0, \rho)$ specified above. This function $U^{d}(T, m, \varepsilon)$ is holomorphic w.r.t $T$ on a domain $\mathcal{R}_{d, \delta} \cap D\left(0, r_{1}\right)$ for any radius $0<r_{1} \leq q^{(1 / 2-\alpha) / k} / 2$ and some $\delta>0$, where

$$
\mathcal{R}_{d, \delta}=\left\{T \in \boldsymbol{C}^{*}|| 1+\frac{e^{i d}}{T} r \mid>\delta, \text { for all } r \geq 0\right\}
$$

continuous w.r.t $m$ on $\boldsymbol{R}$ and holomorphic w.r.t $\varepsilon$ on $D\left(0, \varepsilon_{0}\right)$.

Proof. Under the requirements stated above in Proposition 10, we see that all the assumptions of Proposition 9 are fufilled. As a result, the $q$-Borel transform of order $k$ of the formal series $\hat{U}(T, m, \varepsilon)$ (constructed in Proposition 8) given by $\omega_{k}(\tau, m, \varepsilon)=\sum_{n \geq 0} U_{n}(m, \varepsilon) \tau^{n} /\left(q^{1 / k}\right)^{n(n-1) / 2}$ is convergent with respect to $\tau$ on $D(0, \rho)$ as series in the Banach space $E_{(\beta, \mu)}$. Moreover, this function $\omega_{k}(\tau, m, \varepsilon)$ can be extended as an analytic function with respect to $\tau$ on the unbounded sector $S_{d}$, denoted $\omega_{k}^{d}(\tau, m, \varepsilon)$, that belongs to the Banach space $\mathcal{B} \operatorname{Exp}_{(k, \beta, \mu, \alpha, \rho)}^{q}$ and with the bounds $\left\|\omega_{k}^{d}(\tau, m, \varepsilon)\right\|_{(k, \beta, \mu, \alpha, \rho)} \leq \varpi_{d}$ where $\varpi_{d}$ is some constant independent of $\varepsilon \in D\left(0, \varepsilon_{0}\right)$. From Definition 7 , one can built the $q$-Laplace transform of order $k$ of the function $\tau \mapsto \omega_{k}^{d}(\tau, m, \varepsilon)$ given by the formula (77) which is well defined on the domain described in the statement of Proposition 10. Since $\omega_{k}^{d}(\tau, m, \varepsilon)$ solves the convolution equation (58), we deduce from the commutation rules (40) describes in Proposition 6, that $U^{d}(T, m, \varepsilon)$ actually solves the equation (52) and then also the initial convolution equation (50) we departed from.

\section{Analytic solutions to a $q$-analog of an initial value Cauchy problem with complex parameter}

Let $k \geq 1$ and $D \geq 3$ be integers. Let $q>1$ be a real number. Let $d_{D} \geq 1$ be an integer. For $1 \leq l \leq D-1$, let $d_{l}, \delta_{l} \geq 1$ and $\Delta_{l} \geq 0$ be nonnegative integers. We make the assumption that

$$
1=\delta_{1}, \quad \delta_{l}<\delta_{l+1},
$$

for all $1 \leq l \leq D-2$. We make also the assumption that

$$
\Delta_{l} \geq d_{l}, \quad \frac{d_{l}}{k}+1 \geq \delta_{l}, \quad \frac{d_{D}}{k}+1>\delta_{l}
$$

for all $1 \leq l \leq D-1$. Let $Q(X), R_{l}(X) \in C[X], 1 \leq l \leq D$, be polynomials such that

$$
\operatorname{deg}(Q) \geq \operatorname{deg}\left(R_{D}\right) \geq \operatorname{deg}\left(R_{l}\right), \quad Q(\text { im }) \neq 0, \quad R_{D}(\text { im }) \neq 0
$$


for all $m \in \boldsymbol{R}$, all $1 \leq l \leq D-1$. We consider the following initial value Cauchy problem

$$
\begin{aligned}
Q\left(\partial_{z}\right) \sigma_{q, t} u(t, z, \varepsilon)= & (\varepsilon t)^{d_{D}} \sigma_{q, t}^{d_{D} / k+1} R_{D}\left(\partial_{z}\right) u(t, z, \varepsilon) \\
& +\sum_{l=1}^{D-1} \varepsilon^{\Delta_{l}} t^{d_{l}} \sigma_{q, t}^{\delta_{l}}\left(c_{l}(t, z, \varepsilon) R_{l}\left(\partial_{z}\right) u(t, z, \varepsilon)\right)+\sigma_{q, t} f(t, z, \varepsilon) .
\end{aligned}
$$

The coefficients $c_{l}(t, z, \varepsilon)$ and the forcing term $f(t, z, \varepsilon)$ are constructed as follows. We consider sequences of functions $m \mapsto C_{l, n}(m, \varepsilon)$ and $m \mapsto F_{n}(m, \varepsilon)$ for all $n \geq 0,1 \leq l \leq D-1$ that belong to the Banach space $E_{(\beta, \mu)}$ for some $\beta>0$ and $\mu>\operatorname{deg}\left(R_{D}\right)+1$ and which depend holomorphically on $\varepsilon \in D\left(0, \varepsilon_{0}\right)$. We assume that there exist constants $\tilde{C}_{l}, C_{F}, T_{0}>0$ such that (49) holds for all $1 \leq l \leq D-1$, all $n \geq 0$, all $\varepsilon \in D\left(0, \varepsilon_{0}\right)$. We also take a real number $\alpha \in \boldsymbol{R}$ such that $(53)$ holds. We introduce the functions

$$
\begin{aligned}
& \mathscr{C}_{l}(T, z, \varepsilon)=\sum_{n \geq 0} \mathcal{F}^{-1}\left(m \mapsto C_{l, n}(m, \varepsilon)\right)(z) T^{n}, \\
& \mathscr{F}(T, z, \varepsilon)=\sum_{n \geq 0} \mathcal{F}^{-1}\left(m \mapsto F_{n}(m, \varepsilon)\right)(z) T^{n}
\end{aligned}
$$

which define bounded holomorphic functions on $D\left(0, T_{0} / 2\right) \times H_{\beta^{\prime}} \times D\left(0, \varepsilon_{0}\right)$ for any $0<\beta^{\prime}<\beta$, for all $1 \leq l \leq D-1$ (where $\mathcal{F}^{-1}$ denotes the inverse Fourier transform defined in Proposition 7). We define the coefficients $c_{l}(t, z, \varepsilon), 1 \leq$ $l \leq D-1$ and the forcing term $f(t, z, \varepsilon)$ as

$$
c_{l}(t, z, \varepsilon)=\mathscr{C}_{l}(\varepsilon t, z, \varepsilon), \quad f(t, z, \varepsilon)=\mathscr{F}(\varepsilon t, z, \varepsilon) .
$$

By construction, the functions $c_{l}(t, z, \varepsilon), 1 \leq l \leq D-1$ and $f(t, z, \varepsilon)$ are bounded holomorphic functions on $D(0, r) \times H_{\beta^{\prime}} \times D\left(0, \varepsilon_{0}\right)$ where $r \varepsilon_{0}<T_{0} / 2$.

We make the additional requirement that there exists an unbounded sector

$$
S_{Q, R_{D}}=\left\{z \in C|| z\left|\geq r_{Q, R_{D}},\right| \arg (z)-d_{Q, R_{D}} \mid \leq \eta_{Q, R_{D}}\right\}
$$

with direction $d_{Q, R_{D}} \in \boldsymbol{R}$, aperture $\eta_{Q, R_{D}}>0$ for some radius $r_{Q, R_{D}}>0$ such that

$$
\frac{Q(i m)}{R_{D}(i m)} \in S_{Q, R_{D}}
$$

for all $m \in \boldsymbol{R}$.

Definition 8. Let $\varsigma \geq 2$ be an integer. For all $0 \leq p \leq \varsigma-1$, we consider open sectors $\mathcal{E}_{p}$ centered at 0 , with radius $\varepsilon_{0}$ such that $\mathcal{E}_{p} \cap \mathcal{E}_{p+1} \neq \varnothing$, for all $0 \leq p \leq \varsigma-1$ (with the convention that $\mathcal{E}_{\varsigma}=\mathcal{E}_{0}$ ). Moreover, we assume that 
the intersection of any three different elements in $\left\{\mathcal{E}_{p}\right\}_{0 \leq p \leq \varsigma-1}$ is empty and that $\bigcup_{p=0}^{\varsigma-1} \mathcal{E}_{p}=\mathcal{U} \backslash\{0\}$, where $\mathcal{U}$ is some neighborhood of 0 in $C$. Such a set of sectors $\left\{\mathcal{E}_{p}\right\}_{0 \leq p \leq \varsigma-1}$ is called a good covering in $\boldsymbol{C}^{*}$.

Definition 9. Let $\left\{\mathcal{E}_{p}\right\}_{0 \leq p \leq \varsigma-1}$ be a good covering in $\boldsymbol{C}^{*}$ and let $\mathcal{T}$ be an open bounded sector centered at 0 with radius $r_{\mathcal{T}}>0$. We make the assumption that

$$
0<\varepsilon_{0}<1, \quad 0<r_{\mathcal{T}}<1, \quad \alpha+\frac{k}{\log (q)} \log \left(r_{\mathcal{T}}\right) \leq 0, \quad \varepsilon_{0} r_{\mathcal{T}} \leq q^{(1 / 2-\alpha) / k} / 2
$$

and consider a family of open domains $\mathcal{R}_{\mathfrak{D}_{p}, \delta} \cap D\left(0, \varepsilon_{0} r_{\mathcal{T}}\right)$, where

$$
\mathcal{R}_{\mathfrak{D}_{p}, \delta}=\left\{T \in C^{*}|| 1+\frac{e^{i \mathfrak{D}_{p}}}{T} r \mid>\delta, \text { for all } r \geq 0\right\}
$$

such that $\mathfrak{D}_{p} \in \boldsymbol{R}, 0 \leq p \leq \varsigma-1$ are directions which satisfy the next constraints. Let $q_{l}(m)$ be the roots of the polynomials $P_{m}(\tau)$ given by (62), $S_{\mathfrak{D}_{p}}, 0 \leq p \leq \varsigma-1$, be unbounded sectors centered at 0 with bisecting direction $\mathfrak{D}_{p}$, with small aperture and a disc $D(0, \rho)$ chosen in such a way that the next three constraints hold:

1) There exists a constant $M_{1}>0$ such that

$$
\left|\tau-q_{l}(m)\right| \geq M_{1}(1+|\tau|)
$$

for all $m \in \boldsymbol{R}$, all $\tau \in S_{\mathrm{D}_{p}} \cup \bar{D}(0, \rho)$, all $0 \leq p \leq \varsigma-1$ and all $0 \leq l \leq d_{D}-1$.

2) There exists a constant $M_{2}>0$ with

$$
\left|\tau-q_{l_{0}}(m)\right| \geq M_{2}\left|q_{l_{0}}(m)\right|
$$

for all $m \in \boldsymbol{R}$, all $\tau \in S_{\mathfrak{D}_{p}} \cup \bar{D}(0, \rho)$, all $0 \leq p \leq \varsigma-1$ and some $l_{0} \in\{0, \ldots$, $\left.d_{D}-1\right\}$.

3) For all $0 \leq p \leq \varsigma-1$, all $t \in \mathcal{T}$ and all $\varepsilon \in \mathcal{E}_{p}$, we have that $\varepsilon t \in$ $\mathcal{R}_{\mathfrak{D}_{p}, \delta} \cap D\left(0, \varepsilon_{0} r_{\mathcal{T}}\right)$.

The family $\left\{\left(\mathcal{R}_{\varpi_{p}, \delta}\right)_{0 \leq p \leq \varsigma-1}, D(0, \rho), \mathcal{T}\right\}$ is said to be associated to the good covering $\left\{\mathcal{E}_{p}\right\}_{0 \leq p \leq \varsigma-1}$.

In our next main result, we build a family of actual holomorphic solutions to the equation (81) defined on the sectors $\mathcal{E}_{p}$ with respect to the complex parameter $\varepsilon$. We can also control the difference between any two neighboring solutions on the intersections $\mathcal{E}_{p} \cap \mathcal{E}_{p+1}$ and show that it is $q$-exponential flat of order $k$.

Theorem 1. Assume that the conditions (78), (79), (80) and (83) hold. We take for granted that the coefficients $c_{l}(t, z, \varepsilon)$ and the forcing term $f(t, z, \varepsilon)$ 
are given by the expressions (82). Let $\left\{\mathcal{E}_{p}\right\}_{0 \leq p \leq \varsigma-1}$ be a given good covering in $\boldsymbol{C}^{*}$ for which a family $\left\{\left(\mathcal{R}_{\varpi_{p}, \delta}\right)_{0 \leq p \leq \varsigma-1}, D(0, \rho), \mathcal{T}\right\}$ associated to it can be considered. Then, there exist a radius $r_{Q, R_{D}}>0$ large enough and constants $\zeta_{l}, \zeta_{\psi}>0$ small enough such that if

$$
\tilde{C}_{l} \leq \zeta_{l}, \quad C_{F} \leq \zeta_{\psi}
$$

for all $1 \leq l \leq D-1$, then for every $0 \leq p \leq \varsigma-1$, one can construct a solution $u_{p}(t, z, \varepsilon)$ of (81) which defines a bounded holomorphic function on the domain $\mathcal{T} \times H_{\beta^{\prime}} \times \mathcal{E}_{p}$ for any given $0<\beta^{\prime}<\beta . \quad$ Moreover, there exist constants $K_{p}^{1} \in \boldsymbol{R}$, $K_{p}^{2}>0$ such that

$$
\sup _{t \in \mathcal{T}, z \in H_{\beta^{\prime}}}\left|u_{p+1}(t, z, \varepsilon)-u_{p}(t, z, \varepsilon)\right| \leq K_{p}^{2} \exp \left(-\frac{k}{2 \log (q)} \log ^{2}(|\varepsilon|)\right)|\varepsilon|^{K_{p}^{1}}
$$

for all $\varepsilon \in \mathcal{E}_{p+1} \cap \mathcal{E}_{p}$, for all $0 \leq p \leq \varsigma-1$ (where by convention $u_{\varsigma}=u_{0}$ ).

Proof. From the statement of Proposition 10 and under the hypotheses of Theorem 1, for each direction $\mathfrak{D}_{p}$ chosen as in Definition 9, one can construct a function $U^{\mathfrak{D}_{p}}(T, m, \varepsilon)$ which solves the convolution equation

$$
\begin{aligned}
Q(i m) \sigma_{q, T} & U^{\mathfrak{D}_{p}}(T, m, \varepsilon) \\
= & T^{d_{D}} \sigma_{q, T}^{d_{D} / k+1} R_{D}(i m) U^{\mathfrak{\mho}_{p}}(T, m, \varepsilon)+\sum_{l=1}^{D-1} \varepsilon^{\Delta_{l}-d_{l}} T^{d_{l}} \sigma_{q, T}^{\delta_{l}} \\
& \times\left(\frac{1}{(2 \pi)^{1 / 2}} \int_{-\infty}^{+\infty} C_{l}\left(T, m-m_{1}, \varepsilon\right) R_{l}\left(i m_{1}\right) U^{\mathfrak{\mho}_{p}}\left(T, m_{1}, \varepsilon\right) d m_{1}\right) \\
& +\sigma_{q, T} F(T, m, \varepsilon),
\end{aligned}
$$

where

$$
C_{l}(T, m, \varepsilon)=\sum_{n \geq 0} C_{l, n}(m, \varepsilon) T^{n}, \quad F(T, m, \varepsilon)=\sum_{n \geq 0} F_{n}(m, \varepsilon) T^{n}
$$

are convergent power series on $D\left(0, T_{0} / 2\right)$ with values in $E_{(\beta, \mu)}$, for all $\varepsilon \in D\left(0, \varepsilon_{0}\right)$. This function $U^{\mathfrak{d}_{p}}(T, m, \varepsilon)$ is holomorphic w.r.t $T$ on a domain $\mathcal{R}_{\mathfrak{D}_{p}, \delta} \cap D\left(0, r_{1}\right)$ where $0<r_{1} \leq q^{(1 / 2-\alpha) / k} / 2$ and some $\delta>0$ (as described in Proposition 10). Additionally, $U^{\mathfrak{D}_{p}}(T, m, \varepsilon)$ can be written as a $q$-Laplace transform of order $k$ in direction $\mathfrak{D}_{p}$

$$
U^{\mathfrak{D}_{p}}(T, m, \varepsilon)=\frac{1}{\pi_{q^{1 / k}}} \int_{L_{\gamma_{p}}} \frac{\omega_{k}^{\mathfrak{D}_{p}}(u, m, \varepsilon)}{\Theta_{q^{1 / k}}(u / T)} \frac{d u}{u}
$$


where $L_{\gamma_{p}}=\boldsymbol{R}_{+} e^{i \gamma_{p}} \in S_{\mathfrak{D}_{p}} \cup\{0\}$, where $\omega_{k}^{\mathfrak{D}_{p}}(\tau, m, \varepsilon)$ defines a continuous function on $\left(\bar{D}(0, \rho) \cup \bar{S}_{\mathfrak{D}_{p}}\right) \times \boldsymbol{R} \times D\left(0, \varepsilon_{0}\right)$, holomorphic w.r.t $(\tau, \varepsilon)$ on $\left(D(0, \rho) \cup S_{\mathfrak{D}_{p}}\right) \times$ $D\left(0, \varepsilon_{0}\right)$ for any $m \in \boldsymbol{R}$ and satisfies the next estimates: there exists a constant $\varpi_{\triangleright_{p}}>0$ such that

$$
\left|\omega_{k}^{\mathfrak{D}_{p}}(\tau, m, \varepsilon)\right| \leq \varpi_{\mathfrak{D}_{p}}(1+|m|)^{-\mu} e^{-\beta|m|} \exp \left(\frac{k}{2} \frac{\log ^{2}(|\tau|)}{\log (q)}+\alpha \log |\tau|\right)
$$

for all $\tau \in S_{\mathbb{D}_{p}}$, all $m \in \boldsymbol{R}$, all $\varepsilon \in D\left(0, \varepsilon_{0}\right)$. Moreover, these functions $\tau \mapsto$ $\omega_{k}^{\mathfrak{D}_{p}}(\tau, m, \varepsilon)$ are analytic continuation on the sectors $S_{\mathfrak{\triangleright}_{p}}$ of a common function

$$
\omega_{k}(\tau, m, \varepsilon)=\sum_{n \geq 0} U_{n}(m, \varepsilon) \frac{\tau^{n}}{\left(q^{1 / k}\right)^{n(n-1) / 2}}
$$

which is a convergent power series on $D(0, \rho)$ with coefficients $U_{n}(m, \varepsilon)$ in $E_{(\beta, \mu)}$ with the property that the series $\hat{U}(T, m, \varepsilon)=\sum_{n \geq 0} U_{n}(m, \varepsilon) T^{n}$ formally solves the equation (88) as explained in Proposition 8. Due to the estimates (89) and the assumption (84), one can see that the function

$$
\mathscr{U}^{\mathfrak{D}_{p}}(T, z, \varepsilon)=\mathcal{F}^{-1}\left(m \mapsto U^{\mathfrak{D}_{p}}(T, m, \varepsilon)\right)(z)
$$

defines a bounded holomorphic function on $\left(\mathcal{R}_{\mathfrak{D}_{p}, \delta} \cap D\left(0, \varepsilon_{0} r_{\mathcal{T}}\right)\right) \times H_{\beta^{\prime}} \times D\left(0, \varepsilon_{0}\right)$, for any $0<\beta^{\prime}<\beta$. For all $0 \leq p \leq \varsigma-1$, we define

$$
u_{p}(t, z, \varepsilon)=\mathscr{U}^{\mathfrak{D}_{p}}(\varepsilon t, z, \varepsilon)=\frac{1}{\pi_{q^{1 / k}}(2 \pi)^{1 / 2}} \int_{-\infty}^{+\infty} \int_{L_{\gamma_{p}}} \frac{\omega_{k}^{\mathrm{D}_{p}}(u, m, \varepsilon)}{\Theta_{q^{1 / k}}(u /(\varepsilon t))} e^{i z m} \frac{d u}{u} d m .
$$

From the requirement 3 ) of Definition $9, u_{p}(t, z, \varepsilon)$ defines a bounded holomorphic function on the domain $\mathcal{T} \times H_{\beta^{\prime}} \times \mathcal{E}_{p}$. Moreover, using the properties of the Fourier inverse transform out of Proposition 7 and reminding that $U^{\mathfrak{D}_{p}}(T, m, \varepsilon)$ solves $(88)$, we deduce that $u_{p}(t, z, \varepsilon)$ solves our main problem (81) on $\mathcal{T} \times H_{\beta^{\prime}} \times \mathcal{E}_{p}$.

In the next step we proceed to the proof of the bounds estimates (87). Indeed, let $0 \leq p \leq \varsigma-1$. From the lower bounds estimates (36), we deduce that the function

$$
u \mapsto \frac{\omega_{k}(u, m, \varepsilon)}{\Theta_{q^{1 / k}}(u /(\varepsilon t)) u}
$$

is bounded and holomorphic on a domain $S_{\mathfrak{D}_{p}, \mathrm{D}_{p+1}} \cap D(0, \rho)$ for some sector $S_{\mathfrak{D}_{p}, \mathrm{D}_{p+1}}$ centered at 0 containing the union $S_{\mathfrak{\triangleright}_{p}} \cup S_{\mathfrak{D}_{p+1}}$, for any $\varepsilon \in \mathcal{E}_{p} \cap \mathcal{E}_{p+1}$, $t \in \mathcal{T}$. Hence, the integral of (92) along the union of a segment starting from 0 to $(\rho / 2) e^{i \mathrm{D}_{p+1}}$, an arc of circle with radius $\rho / 2$ which connects $(\rho / 2) e^{i \mathrm{D}_{p+1}}$ and $(\rho / 2) e^{i \mathrm{D}_{p}}$ and finally a segment starting from $(\rho / 2) e^{i \mathrm{D}_{p}}$ to 0 is equal to zero. As a result, we can write the difference $u_{p+1}-u_{p}$ as a sum of three integrals 


$$
\begin{aligned}
u_{p+1}(t, z, \varepsilon)-u_{p}(t, z, \varepsilon) & \frac{1}{\pi_{q^{1 / k}}(2 \pi)^{1 / 2}} \int_{-\infty}^{+\infty} \int_{L_{\rho / 2, \gamma_{p+1}}} \frac{\omega_{k}^{\mathrm{D}_{p+1}}(u, m, \varepsilon)}{\Theta_{q^{1 / k}}(u /(\varepsilon t))} e^{i z m} \frac{d u}{u} d m \\
& -\frac{1}{\pi_{q^{1 / k}}(2 \pi)^{1 / 2}} \int_{-\infty}^{+\infty} \int_{L_{\rho / 2, \gamma_{p}}} \frac{\omega_{k}^{\mathrm{D}_{p}}(u, m, \varepsilon)}{\Theta_{q^{1 / k}}(u /(\varepsilon t))} e^{i z m} \frac{d u}{u} d m \\
& +\frac{1}{\pi_{q^{1 / k}}(2 \pi)^{1 / 2}} \int_{-\infty}^{+\infty} \int_{C_{\rho / 2, \gamma_{p}, \gamma_{p+1}}} \frac{\omega_{k}(u, m, \varepsilon)}{\Theta_{q^{1 / k}}(u /(\varepsilon t))} e^{i z m} \frac{d u}{u} d m,
\end{aligned}
$$

where $L_{\rho / 2, \gamma_{p+1}}=[\rho / 2,+\infty) e^{i \gamma_{p+1}}, L_{\rho / 2, \gamma_{p}}=[\rho / 2,+\infty) e^{i \gamma_{p}}$ and $C_{\rho / 2, \gamma_{p}, \gamma_{p+1}}$ is an arc of circle with radius $\rho / 2$ connecting $(\rho / 2) e^{i \gamma_{p}}$ and $(\rho / 2) e^{i \gamma_{p+1}}$ for a well chosen orientation.

We show estimates for the first integral

$$
I_{1}=\left|\frac{1}{\pi_{q^{1 / k}}(2 \pi)^{1 / 2}} \int_{-\infty}^{+\infty} \int_{L_{\rho / 2, \gamma_{p+1}}} \frac{\omega_{k}^{\mathrm{D}_{p+1}}(u, m, \varepsilon)}{\Theta_{q^{1 / k}}(u /(\varepsilon t))} e^{i z m} \frac{d u}{u} d m\right| .
$$

From the upper bounds (89) and lower bounds (36) estimates, we get

$$
\begin{aligned}
I_{1} \leq & \frac{1}{\pi_{q^{1 / k}}(2 \pi)^{1 / 2}} \int_{-\infty}^{+\infty} \int_{\rho / 2}^{+\infty} \varpi_{\mathfrak{D}_{p+1}}(1+|m|)^{-\mu} e^{-\beta|m|} \\
& \times \frac{\exp \left(\frac{k}{2} \frac{\log ^{2}(r)}{\log (q)}+\alpha \log (r)\right)}{C_{q, k} \delta \exp \left(\frac{k}{2} \frac{\log ^{2}(r /|\varepsilon t|)}{\log (q)}\right)(r /|\varepsilon t|)^{1 / 2}} e^{-m \operatorname{Im}(z)} \frac{d r}{r} d m .
\end{aligned}
$$

We can expand

$$
\begin{aligned}
\exp ( & \left.\frac{k}{2} \frac{\log ^{2}(r)}{\log (q)}+\alpha \log (r)-\frac{k}{2} \frac{\log ^{2}(r /|\varepsilon t|)}{\log (q)}\right) \\
= & \exp \left(\frac{k}{2 \log (q)}\left(-\log ^{2}|\varepsilon|-2 \log |\varepsilon| \log |t|-\log ^{2}|t|\right)\right) \\
& \times r^{\alpha} \exp \left(\frac{k}{\log (q)} \log (r) \log |\varepsilon|+\frac{k}{\log (q)} \log (r) \log |t|\right) .
\end{aligned}
$$

From the assumption that $0<\varepsilon_{0}<1$ and $0<r_{\mathcal{T}}<1$, we deduce that

$$
\begin{aligned}
& \exp \left(-\frac{k}{\log (q)} \log |\varepsilon| \log |t|\right) \leq|\varepsilon|^{-(k / \log (q)) \log \left(r_{T}\right)}, \\
& \exp \left(\frac{k}{\log (q)} \log (r) \log |\varepsilon|\right) \leq|\varepsilon|^{(k / \log (q)) \log (\rho / 2)}
\end{aligned}
$$


for all $t \in \mathcal{T}, \varepsilon \in \mathcal{E}_{p} \cap \mathcal{E}_{p+1}, r \geq \rho / 2$ and

$$
\begin{array}{ll}
\exp \left(\frac{k}{\log (q)} \log (r) \log (|t|)\right) \leq|t|^{(k / \log (q)) \log (\rho / 2)} & \text { if } \rho / 2 \leq r \leq 1, \\
\exp \left(\frac{k}{\log (q)} \log (r) \log (|t|)\right) \leq r^{(k / \log (q)) \log \left(r_{\mathcal{T}}\right)} & \text { if } r \geq 1
\end{array}
$$

for all $t \in \mathcal{T}$. On the other hand, we can check that there exists a constant $K_{k, \rho, q}>0$ with

$$
\sup _{x>0} x^{(k / \log (q)) \log (\rho / 2)} \exp \left(-\frac{k}{2 \log (q)} \log ^{2}(x)\right) \leq K_{k, \rho, q} .
$$

Taking into account the constraints (84) and gathering (96), (97) and (98), we deduce from (95) that there exist two constants $\tilde{K}^{1} \in \boldsymbol{R}$ and $\tilde{K}^{2}>0$ with

$$
\begin{gathered}
\exp \left(\frac{k}{2} \frac{\log ^{2}(r)}{\log (q)}+\alpha \log (r)-\frac{k}{2} \frac{\log ^{2}(r /|\varepsilon t|)}{\log (q)}\right) \\
\leq \tilde{K}^{2} \exp \left(-\frac{k}{2 \log (q)} \log ^{2}|\varepsilon|\right)|\varepsilon|^{\tilde{K}^{1}}
\end{gathered}
$$

for all $t \in \mathcal{T}$, all $r \geq \rho / 2$, all $\varepsilon \in \mathcal{E}_{p} \cap \mathcal{E}_{p+1}$. Hence, from (94) and (99) we deduce that

$$
\begin{aligned}
I_{1} \leq & \frac{\tilde{K}^{2} \varpi_{\mathfrak{D}_{p+1}}\left(\varepsilon_{0} r_{\mathcal{T}}\right)^{1 / 2}}{\pi_{q^{1 / k}}(2 \pi)^{1 / 2} C_{q, k} \delta} \int_{-\infty}^{+\infty} e^{-\left(\beta-\beta^{\prime}\right)|m|} d m \\
& \times \int_{\rho / 2}^{+\infty} \frac{1}{r^{3 / 2}} d r \exp \left(-\frac{k}{2 \log (q)} \log ^{2}|\varepsilon|\right)|\varepsilon|^{\tilde{K}^{1}} \\
\leq & \frac{4 \tilde{K}^{2} \varpi_{\mathfrak{D}_{p+1}}\left(\varepsilon_{0} r_{\mathcal{T}}\right)^{1 / 2}}{\pi_{q^{1 / k}}(2 \pi)^{1 / 2} C_{q, k} \delta\left(\beta-\beta^{\prime}\right)(\rho / 2)^{1 / 2}} \exp \left(-\frac{k}{2 \log (q)} \log ^{2}|\varepsilon|\right)|\varepsilon|^{\tilde{K}^{1}}
\end{aligned}
$$

for all $t \in \mathcal{T}$, all $z \in H_{\beta^{\prime}}$, all $\varepsilon \in \mathcal{E}_{p} \cap \mathcal{E}_{p+1}$.

In a similar manner, we can provide estimates for the second integral

$$
I_{2}=\left|\frac{1}{\pi_{q^{1 / k}}(2 \pi)^{1 / 2}} \int_{-\infty}^{+\infty} \int_{L_{\rho / 2, \gamma_{p}}} \frac{\omega_{k}^{\mathbb{D}_{p}}(u, m, \varepsilon)}{\Theta_{q^{1 / k}}(u /(\varepsilon t))} e^{i z m} \frac{d u}{u} d m\right| .
$$

Indeed, again from the $q$-exponential growth estimates (89) and following the same steps as above yields

$$
I_{2} \leq \frac{4 \tilde{K}^{2} \varpi_{\mathfrak{D}_{p}}\left(\varepsilon_{0} r_{\mathcal{T}}\right)^{1 / 2}}{\pi_{q^{1 / k}}(2 \pi)^{1 / 2} C_{q, k} \delta\left(\beta-\beta^{\prime}\right)(\rho / 2)^{1 / 2}} \exp \left(-\frac{k}{2 \log (q)} \log ^{2}|\varepsilon|\right)|\varepsilon|^{\tilde{K}^{1}}
$$

for all $t \in \mathcal{T}$, all $z \in H_{\beta^{\prime}}$, all $\varepsilon \in \mathcal{E}_{p} \cap \mathcal{E}_{p+1}$. 
In the final step, we need to give estimates for the last integral

$$
I_{3}=\left|\frac{1}{\pi_{q^{1 / k}}(2 \pi)^{1 / 2}} \int_{-\infty}^{+\infty} \int_{C_{\rho / 2, \gamma_{p}, \gamma_{p+1}}} \frac{\omega_{k}(u, m, \varepsilon)}{\Theta_{q^{1 / k}}(u /(\varepsilon t))} e^{i z m} \frac{d u}{u} d m\right| .
$$

By construction of $\omega_{k}(u, m, \varepsilon)$ in (90) and the lower bounds (36) estimates, we deduce the existence of a constant $\varpi>0$ with

$$
I_{3} \leq \frac{1}{\pi_{q^{1 / k}}(2 \pi)^{1 / 2}} \int_{-\infty}^{+\infty}\left|\int_{\gamma_{p}}^{\gamma_{p+1}} \frac{\varpi(1+|m|)^{-\mu} e^{-\beta|m|}}{C_{q, k} \delta \exp \left(\frac{k}{2} \frac{\log ^{2}(\rho /(2|\varepsilon t|))}{\log (q)}\right)\left(\frac{\rho}{2|\varepsilon t|}\right)^{1 / 2}} e^{-m \operatorname{Im}(z)} d \theta\right| d m
$$

As above, we can expand

$$
\begin{aligned}
\exp (- & \left.\frac{k}{2} \frac{\log ^{2}(\rho /(2|\varepsilon t|))}{\log (q)}\right) \\
= & \exp \left(-\frac{k}{2 \log (q)} \log ^{2}\left(\frac{\rho}{2}\right)\right)|\varepsilon|^{(k / \log (q)) \log (\rho / 2)}|t|^{(k / \log (q)) \log (\rho / 2)} \\
& \times \exp \left(\frac{k}{2 \log (q)}\left(-\log ^{2}|\varepsilon|-2 \log (|\varepsilon|) \log (|t|)-\log ^{2}(|t|)\right)\right) .
\end{aligned}
$$

From the assumption $0<\varepsilon_{0}<1$, we check that

$$
\exp \left(-\frac{k}{\log (q)} \log (|\varepsilon|) \log (|t|)\right) \leq|\varepsilon|^{-(k / \log (q)) \log \left(r_{\mathcal{T}}\right)}
$$

for all $t \in \mathcal{T}$, all $\varepsilon \in \mathcal{E}_{p} \cap \mathcal{E}_{p+1}$. Gathering (104) and (98), we deduce from (103) two constants $\hat{K}^{1} \in \boldsymbol{R}$ and $\hat{K}^{2}>0$ such that

$$
\exp \left(-\frac{k}{2} \frac{\log ^{2}(\rho /(2|\varepsilon t|))}{\log (q)}\right) \leq \hat{K}^{2} \exp \left(-\frac{k}{2 \log (q)} \log ^{2}|\varepsilon|\right)|\varepsilon|^{\hat{K}^{1}}
$$

for all $t \in \mathcal{T}$, all $\varepsilon \in \mathcal{E}_{p} \cap \mathcal{E}_{p+1}$. From (102) and (105) we deduce that

$$
\begin{aligned}
I_{3} \leq & \frac{\varpi\left|\gamma_{p+1}-\gamma_{p}\right| \hat{K}^{2}\left(\varepsilon_{0} r_{\mathcal{T}}\right)^{1 / 2}}{\pi_{q^{1 / k}}(2 \pi)^{1 / 2} C_{q, k} \delta(\rho / 2)^{1 / 2}} \int_{-\infty}^{+\infty} e^{-\left(\beta-\beta^{\prime}\right)|m|} d m \\
& \times \exp \left(-\frac{k}{2 \log (q)} \log ^{2}|\varepsilon|\right)|\varepsilon|^{\hat{K}^{1}} \\
\leq & \frac{2 \varpi\left|\gamma_{p+1}-\gamma_{p}\right| \hat{K}^{2}\left(\varepsilon_{0} r_{\mathcal{T}}\right)^{1 / 2}}{\pi_{q^{1 / k}}(2 \pi)^{1 / 2} C_{q, k} \delta(\rho / 2)^{1 / 2}\left(\beta-\beta^{\prime}\right)} \exp \left(-\frac{k}{2 \log (q)} \log ^{2}|\varepsilon|\right)|\varepsilon|^{\hat{K}^{1}}
\end{aligned}
$$

for all $t \in \mathcal{T}$, all $z \in H_{\beta^{\prime}}$, all $\varepsilon \in \mathcal{E}_{p} \cap \mathcal{E}_{p+1}$. 
Finally, gathering the estimates (100), (101) and (106), we deduce from the decomposition (93) the promised estimates (87).

\section{6. $q$-Gevrey asymptotics of order $1 / k$ for the analytic solutions to the initial value Cauchy problem}

\subsection{Holomorphic functions on sectors with $q$-Gevrey asymptotic expansion}

The following characterization is a slight modification of the Definition 5.1 given in [8].

Definition 10. Let $V$ be a bounded open sector centered at 0 in $C$. Let $\left(\boldsymbol{F},\|.\|_{\boldsymbol{F}}\right)$ be a complex Banach space. Let $q>1$ be a real number and $k \geq 1$ be an integer. We say that a holomorphic function $f: V \rightarrow \boldsymbol{F}$ admits a formal series $\hat{f}(\varepsilon)=\sum_{n \geq 0} f_{n} \varepsilon^{n} \in \boldsymbol{F}[[\varepsilon]]$ as its $q$-Gevrey asymptotic expansion of order $1 / k$ if for every open subsector $U \subset V$ with $\bar{U} \subset V$, there exist constants $A, C>0$ such that

$$
\left\|f(\varepsilon)-\sum_{n=0}^{N} f_{n} \varepsilon^{n}\right\|_{\boldsymbol{F}} \leq C A^{N+1} q^{(N+1) N /(2 k)}|\varepsilon|^{N+1}
$$

for all $\varepsilon \in U$, all $N \geq 0$.

The next lemma is a reshaped version of Proposition 5.3 stated in [8]. This result is a $q$-analog of the well known statement that a holomorphic function is exponentially flat of order $k$ on a sector $S$ if and only if its has $\hat{0}$ as asymptotic expansion of Gevrey order $1 / k$ on $S$, see [7], Theorem XI-3-2.

Lemma 7. A holomorphic function $f: V \rightarrow \boldsymbol{F}$ admits the null formal series $\hat{0} \in \boldsymbol{F}[\varepsilon]]$ as its q-Gevrey asymptotic expansion of order $1 / k$ if and only for any open subsector $U \subset V$ with $\bar{U} \subset V$ there exist two constants $K_{1} \in \boldsymbol{R}, K_{2}>0$ with

$$
\|f(\varepsilon)\|_{\boldsymbol{F}} \leq K_{2} \exp \left(-\frac{k}{2 \log (q)} \log ^{2}|\varepsilon|\right)|\varepsilon|^{K_{1}}
$$

for all $\varepsilon \in U$.

Proof. Let $A, C>0$ be positive constants. We consider the functions

$$
\begin{aligned}
& G(x)=C A^{x+1} q^{(x+1) x /(2 k)}|\varepsilon|^{x+1}=C \exp (H(x)), \\
& H(x)=(x+1) \log (A)+\frac{\log (q)}{2 k}\left(x^{2}+x\right)+(x+1) \log |\varepsilon|
\end{aligned}
$$

We need to compute $\min _{x \in \boldsymbol{R}} G(x)$. We see that $G(x)$ reaches its minimum where $H(x)$ gets its minimum. An easy computation shows that $H(x)$ gets its 
minimum at $x_{0}=-(k / \log (q)) \log |\varepsilon|+(-(k / \log (q)) \log (A)-1 / 2)$ and we can observe that

(108) $\min _{x \in \boldsymbol{R}} G(x)=G\left(x_{0}\right)=L \exp \left(-\frac{k}{2 \log (q)} \log ^{2}|\varepsilon|\right)|\varepsilon|^{-(k / \log (q)) \log (A)+1 / 2}$,

where $L>0$ is a constant independent of $\varepsilon$. One can check that the lemma is a consequence of (108) using standard computations.

\subsection{A $q$-analog of the Ramis-Sibuya theorem}

The classical Ramis-Sibuya theorem is a cohomological criterion that ensures the $k$-summability of a given formal series (see [2], Section 4.4 Proposition 2 or [7], Lemma XI-2-6). In this subsection, we present a version of this theorem in the framework of $q$-Gevrey asymptotics of order $1 / k$. Its statement is very much alike Theorem 25 given in [10] by A. Lastra and the author. For the sake of clarity for the reader, we give a thorough proof of the result although being very similar to the one given in [10].

Theorem (q-RS). Let $\left(\boldsymbol{F},\|\cdot\|_{\boldsymbol{F}}\right)$ be a Banach space and $\left\{\mathcal{E}_{p}\right\}_{0 \leq p \leq \varsigma-1}$ be a good covering in $\boldsymbol{C}^{*}$. For all $0 \leq p \leq \varsigma-1$, let $G_{p}(\varepsilon)$ be a holomorphic function from $\mathcal{E}_{p}$ into $\boldsymbol{F}$ and let the cocycle $\Delta_{p}(\varepsilon)=G_{p+1}(\varepsilon)-G_{p}(\varepsilon)$ be a holomorphic function from $Z_{p}=\mathcal{E}_{p+1} \cap \mathcal{E}_{p}$ into $\boldsymbol{F}$ (with the convention that $\mathcal{E}_{\zeta}=\mathcal{E}_{0}$ and $\left.G_{\varsigma}=G_{0}\right)$. We make further assumptions.

1) The functions $G_{p}(\varepsilon)$ are bounded as $\varepsilon$ tends to 0 on $\mathcal{E}_{p}$, for all $0 \leq$ $p \leq \varsigma-1$.

2) The function $\Delta_{p}(\varepsilon)$ is q-exponentially flat of order $k$ on $Z_{p}$ for all $0 \leq$ $p \leq \varsigma-1$, meaning that there exist two constants $C_{p}^{1} \in \boldsymbol{R}$ and $C_{p}^{2}>0$ with

$$
\left\|\Delta_{p}(\varepsilon)\right\|_{\boldsymbol{F}} \leq C_{p}^{2}|\varepsilon|^{C_{p}^{1}} \exp \left(-\frac{k}{2 \log (q)} \log ^{2}|\varepsilon|\right)
$$

for all $\varepsilon \in Z_{p}$, all $0 \leq p \leq \varsigma-1$.

Then, there exists a formal power series $\hat{\boldsymbol{G}}(\varepsilon) \in \boldsymbol{F}[[\varepsilon]]$ which is the common $q$-Gevrey asymptotic expansion of order $1 / k$ of the functions $G_{p}(\varepsilon)$ on $\mathcal{E}_{p}$, for all $0 \leq p \leq \varsigma-1$.

Proof. We first start with an essential lemma.

Lemma 8. For all $0 \leq l \leq \varsigma-1$, there exist bounded holomorphic functions $\Psi_{l}: \mathcal{E}_{l} \rightarrow C$ such that

$$
\Delta_{l}(\varepsilon)=\Psi_{l+1}(\varepsilon)-\Psi_{l}(\varepsilon)
$$


for all $\varepsilon \in Z_{l}$, where by convention $\Psi_{\varsigma}(\varepsilon)=\Psi_{0}(\varepsilon)$. Moreover, there exist coefficients $\varphi_{m} \in \boldsymbol{F}, m \geq 0$, such that for each $0 \leq l \leq \varsigma-1$ and any closed proper subsector $\mathcal{W} \subset \mathcal{E}_{l}$, centered at 0 , there exist two constants $\hat{K}_{l}, \hat{M}_{l}>0$ with

$$
\left\|\Psi_{l}(\varepsilon)-\sum_{m=0}^{M} \varphi_{m} \varepsilon^{m}\right\|_{\boldsymbol{F}} \leq \hat{K}_{l}\left(\hat{M}_{l}\right)^{M+1} q^{(M+1) M /(2 k)}|\varepsilon|^{M+1}
$$

for all $\varepsilon \in \mathcal{W}$, all $M \geq 0$.

Proof. We will be devoted to the same arguments as in Lemma XI-2-6 from [7] with suitable modifications in the asymptotic expansions of the functions constructed with the help of the Cauchy-Heine transform. For all $0 \leq l \leq \varsigma-1$, we choose a segment

$$
\mathcal{C}_{l}=\left\{t e^{\sqrt{-1} \theta_{l}} \mid t \in[0, r]\right\} \subset \mathcal{E}_{l} \cap \mathcal{E}_{l+1} .
$$

These $\varsigma$ segments divide the open punctured disc $D(0, r) \backslash\{0\}$ into $\varsigma$ open sectors $\tilde{\mathcal{E}}_{0}, \ldots, \tilde{\mathcal{E}}_{\varsigma-1}$ where

$$
\tilde{\mathcal{E}}_{l}=\left\{\varepsilon \in C^{*}\left|\theta_{l-1}<\arg (\varepsilon)<\theta_{l},\right| \varepsilon \mid<r\right\}, \quad 0 \leq l \leq \varsigma-1,
$$

where by convention $\theta_{-1}=\theta_{\varsigma-1}$. Let

$$
\Psi_{l}(\varepsilon)=\frac{-1}{2 \pi \sqrt{-1}} \sum_{h=0}^{\varsigma-1} \int_{C_{h}} \frac{\Delta_{h}(\xi)}{\xi-\varepsilon} d \xi
$$

for all $\varepsilon \in \tilde{\mathcal{E}}_{l}$, for $0 \leq l \leq \varsigma-1$, be defined as a sum of Cauchy-Heine transforms of the functions $\Delta_{h}(\varepsilon)$. By deformation of the paths $C_{l-1}$ and $C_{l}$ without moving their endpoints and letting the other paths $C_{h}, h \neq l-1, l$ untouched (with the convention that $C_{-1}=C_{\varsigma-1}$ ), one can continue analytically the function $\Psi_{l}$ onto $\mathcal{E}_{l}$. Therefore, $\Psi_{l}$ defines a holomorphic function on $\mathcal{E}_{l}$, for all $0 \leq l \leq \varsigma-1$.

Now, take $\varepsilon \in \mathcal{E}_{l} \cap \mathcal{E}_{l+1}$. In order to compute $\Psi_{l+1}(\varepsilon)-\Psi_{l}(\varepsilon)$, we write

$$
\begin{aligned}
& \Psi_{l}(\varepsilon)=\frac{-1}{2 \pi \sqrt{-1}} \int_{\hat{C}_{l}} \frac{\Delta_{l}(\xi)}{\xi-\varepsilon} d \xi+\frac{-1}{2 \pi \sqrt{-1}} \sum_{h=0, h \neq l}^{\zeta-1} \int_{C_{h}} \frac{\Delta_{h}(\xi)}{\xi-\varepsilon} d \xi \\
& \Psi_{l+1}(\varepsilon)=\frac{-1}{2 \pi \sqrt{-1}} \int_{\check{C}_{l}} \frac{\Delta_{l}(\xi)}{\xi-\varepsilon} d \xi+\frac{-1}{2 \pi \sqrt{-1}} \sum_{h=0, h \neq l}^{\zeta-1} \int_{C_{h}} \frac{\Delta_{h}(\xi)}{\xi-\varepsilon} d \xi
\end{aligned}
$$

where the paths $\hat{C}_{l}$ and $\check{C}_{l}$ are obtained by deforming the same path $C_{l}$ without moving its endpoints in such a way that: 
(a) $\hat{C}_{l} \subset \mathcal{E}_{l} \cap \mathcal{E}_{l+1}$ and $\check{C}_{l} \subset \mathcal{E}_{l} \cap \mathcal{E}_{l+1}$,

(b) $\Gamma_{l, l+1}:=-\check{C}_{l}+\hat{C}_{l}$ is a simple closed curve with positive orientation whose interior contains $\varepsilon$.

Therefore, due to the residue formula, we can write

$$
\Psi_{l+1}(\varepsilon)-\Psi_{l}(\varepsilon)=\frac{1}{2 \pi \sqrt{-1}} \int_{\Gamma_{l, l+1}} \frac{\Delta_{l}(\xi)}{\xi-\varepsilon} d \xi=\Delta_{l}(\varepsilon)
$$

for all $\varepsilon \in \mathcal{E}_{l} \cap \mathcal{E}_{l+1}$, for all $0 \leq l \leq \varsigma-1$ (with the convention that $\Psi_{\varsigma}=\Psi_{0}$ ).

In a second step, we derive asymptotic properties of $\Psi_{l}$. We fix an $0 \leq l \leq \varsigma-1$ and a proper closed sector $\mathcal{W}$ contained in $\mathcal{E}_{l}$. Let $\tilde{C}_{l}$ (resp. $\tilde{C}_{l-1}$ ) be a path obtained by deforming $C_{l}$ (resp. $C_{l-1}$ ) without moving the endpoints in order that $\mathcal{W}$ is contained in the interior of the simple closed curve $\tilde{C}_{l-1}+\gamma_{l}-\tilde{C}_{l}$ (which is itself contained in $\mathcal{E}_{l}$ ), where $\gamma_{l}$ is a circular arc joining the two points $r e^{\sqrt{-1} \theta_{l-1}}$ and $r e^{\sqrt{-1} \theta_{l}}$. We get the representation

$$
\begin{aligned}
\Psi_{l}(\varepsilon)= & \frac{-1}{2 \pi \sqrt{-1}} \int_{\tilde{C}_{l}} \frac{\Delta_{l}(\xi)}{\xi-\varepsilon} d \xi+\frac{-1}{2 \pi \sqrt{-1}} \int_{\tilde{C}_{l-1}} \frac{\Delta_{l-1}(\xi)}{\xi-\varepsilon} d \xi \\
& +\frac{-1}{2 \pi \sqrt{-1}} \sum_{h=0, h \neq l, l-1}^{\zeta-1} \int_{C_{h}} \frac{\Delta_{h}(\xi)}{\xi-\varepsilon} d \xi
\end{aligned}
$$

for all $\varepsilon \in \mathcal{W}$. One assumes that the path $\tilde{C}_{l}$ is given as the union of a segment $L_{l}=\left\{t e^{\sqrt{-1} w_{l}} \mid t \in\left[0, r_{1}\right]\right\}$ where $r_{1}<r$ and $w_{l}>\theta_{l}$ and a curve $\Gamma_{l}=$ $\left\{\mu_{l}(\tau) \mid \tau \in[0,1]\right\}$ such that $\mu_{l}(0)=r_{1} e^{\sqrt{-1} w_{l}}, \mu_{l}(1)=r e^{\sqrt{-1} \theta_{l}}$ and $r_{1} \leq\left|\mu_{l}(\tau)\right|<r$ for all $\tau \in[0,1)$. We also assume that there exists a positive number $\sigma<1$ with $|\varepsilon| \leq \sigma r_{1}$ for all $\varepsilon \in \mathcal{W}$. By construction of the path $\Gamma_{l}$, we get that the function $\varepsilon \mapsto(2 \pi \sqrt{-1})^{-1} \int_{\Gamma_{l}} \Delta_{l}(\xi) /(\xi-\varepsilon) d \xi$ defines an analytic function on the open disc $D\left(0, r_{1}\right)$.

It remains to give estimates for the integral $(2 \pi \sqrt{-1})^{-1} \int_{L_{l}} \Delta_{l}(\xi) /(\xi-\varepsilon) d \xi$. Let $M \geq 0$ be an integer. From the usual geometric series expansion, one can write

$$
\frac{1}{2 \pi \sqrt{-1}} \int_{L_{l}} \frac{\Delta_{l}(\xi)}{\xi-\varepsilon} d \xi=\sum_{m=0}^{M} \alpha_{l, m} \varepsilon^{m}+\varepsilon^{M+1} E_{l, M+1}(\varepsilon),
$$

where

$$
\alpha_{l, m}=\frac{1}{2 \pi \sqrt{-1}} \int_{L_{l}} \frac{\Delta_{l}(\xi)}{\xi^{m+1}} d \xi, \quad E_{l, M+1}(\varepsilon)=\frac{1}{2 \pi \sqrt{-1}} \int_{L_{l}} \frac{\Delta_{l}(\xi)}{\xi^{M+1}(\xi-\varepsilon)} d \xi
$$

for all $\varepsilon \in \mathcal{W}$. 
Gathering (109) and (116), we get that

$$
\left\|\alpha_{l, m}\right\|_{\boldsymbol{F}} \leq \frac{C_{l}^{2}}{2 \pi} \int_{0}^{r_{1}} \frac{\tau^{C_{l}^{1}} \exp \left(-\frac{k}{2 \log (q)} \log ^{2}(\tau)\right)}{\tau^{m+1}} d \tau .
$$

We make the change of variable $t=(k /(2 \log (q)))^{1 / 2} \log (\tau)$ into the right handside of (117) which yields

$$
\begin{aligned}
\frac{C_{l}^{2}}{2 \pi} \int_{0}^{r_{1}} & \frac{\tau^{C_{l}^{1}} \exp \left(-\frac{k}{2 \log (q)} \log ^{2}(\tau)\right)}{\tau^{m+1}} d \tau \\
= & \frac{C_{l}^{2}\left(\frac{2 \log (q)}{k}\right)^{1 / 2}}{2 \pi} \int_{-\infty}^{(k /(2 \log (q)))^{1 / 2} \log \left(r_{1}\right)} \\
& \times \exp \left(\left(\frac{2 \log (q)}{k}\right)^{1 / 2}\left(C_{l}^{1}-m\right) t-t^{2}\right) d t .
\end{aligned}
$$

The use of the Gaussian identity

$$
e^{a^{2} / 4} \sqrt{\pi}=\int_{-\infty}^{+\infty} e^{-x^{2}-a x} d x
$$

for every $a \in \boldsymbol{R}$ (see for instance [1], Chapter 10, p. 498) together with (117) and (118) helps us to get the estimates

$$
\begin{aligned}
\left\|\alpha_{l, m}\right\|_{\boldsymbol{F}} & \leq \frac{C_{l}^{2}\left(\frac{2 \log (q)}{k}\right)^{1 / 2}}{2 \pi^{1 / 2}} \exp \left(\frac{\log (q)}{2 k}\left(C_{l}^{1}-m\right)^{2}\right) \\
& =\frac{C_{l}^{2}\left(\frac{2 \log (q)}{k}\right)^{1 / 2}}{2 \pi^{1 / 2}} q^{\left(C_{l}^{1}\right)^{2} /(2 k)} q^{m\left(-C_{l}^{1} / k-1 /(2 k)\right)} q^{(m+1) m /(2 k)} .
\end{aligned}
$$

On the other hand, one can choose a positive number $\eta>0$ (depending on $\mathcal{W}$ ) such that $|\xi-\varepsilon| \geq|\xi| \sin (\eta)$ for all $\xi \in L_{l}$ and all $\varepsilon \in \mathcal{W}$. Hence, using the same computations as above, we get

$$
\begin{aligned}
\left\|E_{l, M+1}(\varepsilon)\right\|_{\boldsymbol{F}} & \leq \frac{C_{l}^{2}}{2 \pi \sin (\eta)} \int_{0}^{r_{1}} \frac{\tau_{l}^{C_{l}^{1}} \exp \left(-\frac{k}{2 \log (q)} \log ^{2}(\tau)\right)}{\tau^{M+2}} d \tau \\
& \leq \frac{C_{l}^{2}\left(\frac{2 \log (q)}{k}\right)^{1 / 2}}{2 \pi^{1 / 2} \sin (\eta)} \exp \left(\frac{\log (q)}{2 k}\left(C_{l}^{1}-M-1\right)^{2}\right) \\
& =\frac{C_{l}^{2}\left(\frac{2 \log (q)}{k}\right)^{1 / 2}}{2 \pi^{1 / 2} \sin (\eta)} q^{\left(C_{l}^{1}-1\right)^{2} /(2 k)} q^{M\left(-\left(C_{l}^{1}-1\right) / k-1 /(2 k)\right)} q^{(M+1) M /(2 k)}
\end{aligned}
$$

for all $\varepsilon \in \mathcal{W}$. 
Using comparable arguments, one can give estimates of the form (115), (116), (119) and (120) for the other integrals

$$
\frac{-1}{2 \pi \sqrt{-1}} \int_{\tilde{C}_{l-1}} \frac{\Delta_{l-1}(\xi)}{\xi-\varepsilon} d \xi, \quad \frac{-1}{2 \pi \sqrt{-1}} \int_{C_{h}} \frac{\Delta_{h}(\xi)}{\xi-\varepsilon} d \xi
$$

for all $h \neq l, l-1$.

As a consequence, for any $0 \leq l \leq \varsigma-1$, there exist coefficients $\varphi_{l, m} \in \boldsymbol{F}$, $m \geq 0$ and two constants $\hat{K}_{l}, \hat{M}_{l}>0$ such that

$$
\left\|\Psi_{l}(\varepsilon)-\sum_{m=0}^{M} \varphi_{l, m} \varepsilon^{m}\right\|_{\boldsymbol{F}} \leq \hat{K}_{l}\left(\hat{M}_{l}\right)^{M+1} q^{(M+1) M /(2 k)}|\varepsilon|^{M+1}
$$

for all $M \geq 0$, all $\varepsilon \in \mathcal{W}$.

From (109) and (113), we have in particular that there exist two constants $C_{l}^{1} \in \boldsymbol{R}$ and $C_{l}^{2}>0$ with

$$
\left\|\Psi_{l+1}(\varepsilon)-\Psi_{l}(\varepsilon)\right\|_{\boldsymbol{F}} \leq C_{l}^{2}|\varepsilon|^{C_{l}^{1}} \exp \left(-\frac{k}{2 \log (q)} \log ^{2}|\varepsilon|\right)
$$

for all $\varepsilon \in \mathcal{E}_{l+1} \cap \mathcal{E}_{l}$, all $0 \leq l \leq \varsigma-1$. From Lemma 7, we deduce that $\Psi_{l+1}(\varepsilon)-\Psi_{l}(\varepsilon)$ has the formal series $\hat{0}$ as $q$-Gevrey asymptotic expansion of order $1 / k$. From the unicity of the asymptotic expansions on sectors, we deduce that all the formal series $\sum_{m \geq 0} \varphi_{l, m} \varepsilon^{m}, 0 \leq l \leq \varsigma-1$, are equal to some formal series denoted $\hat{\boldsymbol{G}}(\varepsilon)=\sum_{m \geq 0} \varphi_{m} \varepsilon^{m} \in \boldsymbol{F}[[\varepsilon]]$. The Lemma 8 follows.

We consider now the bounded holomorphic functions

$$
a_{i}(\varepsilon)=G_{i}(\varepsilon)-\Psi_{i}(\varepsilon)
$$

for all $0 \leq i \leq \varsigma-1$, all $\varepsilon \in \mathcal{E}_{i}$. By definition, for any $i \in\{0, \ldots, \varsigma-1\}$, we have that

$$
a_{i+1}(\varepsilon)-a_{i}(\varepsilon)=G_{i+1}(\varepsilon)-G_{i}(\varepsilon)-\Delta_{i}(\varepsilon)=0
$$

for all $\varepsilon \in Z_{i}$. Therefore, each $a_{i}(\varepsilon)$ is the restriction on $\mathcal{E}_{i}$ of a holomorphic function $a(\varepsilon)$ on $D(0, r) \backslash\{0\}$. Since $a(\varepsilon)$ is moreover bounded on $D(0, r) \backslash\{0\}$, the origin turns out to be a removable singularity for $a(\varepsilon)$ which, as a consequence, defines a convergent power series on $D(0, r)$.

Finally, one can write

$$
G_{i}(\varepsilon)=a(\varepsilon)+\Psi_{i}(\varepsilon)
$$

for all $\varepsilon \in \mathcal{E}_{i}$, all $0 \leq i \leq \varsigma-1$. Moreover, $a(\varepsilon)$ is a convergent power series, and $\Psi_{i}(\varepsilon)$ has the series $\hat{G}(\varepsilon)=\sum_{m \geq 0} \varphi_{m} \varepsilon^{m}$ as $q$-Gevrey asymptotic expansion of order $1 / k$ on $\mathcal{E}_{i}$, for all $0 \leq i \leq \varsigma-1$. 


\section{3. $q$-Gevrey asymptotic expansion of order $1 / k$ for the analytic solutions constructed in Section 5}

In this subsection, we describe the second main result of our work, namely the construction of a formal power series in the complex parameter $\varepsilon$ whose coefficients are bounded holomorphic functions on the product of a sector with small radius centered at 0 and a strip in $C^{2}$ which formally solves our main problem (81) and which provides a common $q$-Gevrey asymptotic expansion of order $1 / k$ of the actual solutions $u_{p}(t, z, \varepsilon)$ of (81) obtained in Theorem 1 .

The second main outcome of this work reads as follows.

Theorem 2. We take for granted that all the assumptions of Theorem 1 hold. Then, we can construct a formal power series

$$
\hat{u}(t, z, \varepsilon)=\sum_{m \geq 0} h_{m}(t, z) \frac{\varepsilon^{m}}{m !}
$$

solution of (81) with coefficients $h_{m}(t, z)$ that belong to the Banach space $\boldsymbol{F}$ of bounded holomorphic functions on $\mathcal{T} \times H_{\beta^{\prime}}$ endowed with the sup norm and which is the common q-Gevrey asymptotic expansion of order $1 / k$ of the solutions $u_{p}(t, z, \varepsilon)$ of $(81)$ viewed as holomorphic functions from $\mathcal{E}_{p}$ into $\boldsymbol{F}$, for all $0 \leq p \leq \varsigma-1$. Namely, for all $0 \leq p \leq \varsigma-1$, there exists two constants $A_{p}, C_{p}>0$ such that

$$
\sup _{t \in \mathcal{T}, z \in H_{\beta^{\prime}}}\left|u_{p}(t, z, \varepsilon)-\sum_{m=0}^{n} h_{m}(t, z) \frac{\varepsilon^{m}}{m !}\right| \leq C_{p} A_{p}^{n+1} q^{(n+1) n /(2 k)}|\varepsilon|^{n+1}
$$

for all $n \geq 0$, all $\varepsilon \in \mathcal{E}_{p}$.

Proof. We deal with the family of functions $u_{p}(t, z, \varepsilon), 0 \leq p \leq \varsigma-1$ constructed in Theorem 1. For all $0 \leq p \leq \varsigma-1$, we define $G_{p}(\varepsilon):=(t, z)$ $\mapsto u_{p}(t, z, \varepsilon)$, which is by construction a holomorphic and bounded function from $\mathcal{E}_{p}$ into the Banach space $\boldsymbol{F}$ of bounded holomorphic functions on $\mathcal{T} \times H_{\beta^{\prime}}$ equipped with the sup norm, where $\mathcal{T}$ is introduced in Definition 9 and $\beta^{\prime}>0$ is the width of the strip $H_{\beta^{\prime}}$ on which the coefficients $c_{l}(t, z, \varepsilon)$ and forcing term $f(t, z, \varepsilon)$ are holomorphic bounded with respect to $z$ (see (82)). Bearing in mind the estimates (87), we see that the cocycle $\Theta_{p}(\varepsilon)=G_{p+1}(\varepsilon)-G_{p}(\varepsilon)$ is $q$-exponentially flat of order $k$ on $Z_{p}=\mathcal{E}_{p} \cap \mathcal{E}_{p+1}$, for any $0 \leq p \leq \varsigma-1$.

Regarding Theorem $(q-\mathrm{RS})$ stated in subsection 6.2 , we deduce the existence of a formal power series $\hat{\boldsymbol{G}}(\varepsilon) \in \boldsymbol{F}[[\varepsilon]]$ such that the functions $G_{p}(\varepsilon)$ admit $\hat{\boldsymbol{G}}(\varepsilon)$ as their $q$-Gevrey asymptotic expansion of order $1 / k$ on $\mathcal{E}_{p}$, for all $0 \leq p \leq \varsigma-1$. We put

$$
\hat{G}(\varepsilon)=\sum_{m \geq 0} h_{m}(t, z) \varepsilon^{m} / m !=: \hat{u}(t, z, \varepsilon)
$$


In the last part of the proof we show that the formal series $\hat{u}(t, z, \varepsilon)$ solves the main equation $(81)$. Since $\hat{G}(\varepsilon)$ is the asymptotic expansion of the functions $G_{p}(\varepsilon)$ on $\mathcal{E}_{p}$, we get in particular that

$$
\lim _{\varepsilon \rightarrow 0, \varepsilon \in \mathcal{E}_{p}} \sup _{t \in \mathcal{T}, z \in H_{\beta^{\prime}}}\left|\partial_{\varepsilon}^{m} u_{p}(t, z, \varepsilon)-h_{m}(t, z)\right|=0
$$

for all $0 \leq p \leq \varsigma-1$, all $m \geq 0$. Now, we choose some $p \in\{0, \ldots, \varsigma-1\}$. By construction, the function $u_{p}(t, z, \varepsilon)$ is a solution of (81). We take the derivative of order $m \geq 0$ with respect to $\varepsilon$ on the left and right handside of the equation (81). Using Leibniz rule, we deduce that $\partial_{\varepsilon}^{m} u_{p}(t, z, \varepsilon)$ solves the following equation

$$
\begin{aligned}
Q\left(\partial_{z}\right) \sigma_{q, t}\left(\partial_{\varepsilon}^{m} u_{p}\right)(t, z, \varepsilon) & \\
= & \sum_{m_{1}+m_{2}=m} \frac{m !}{m_{1} ! m_{2} !} \partial_{\varepsilon}^{m_{1}}\left(\varepsilon^{d_{D}}\right) t^{d_{D}} \sigma_{q, t}^{d_{D} / k+1} R_{D}\left(\partial_{z}\right)\left(\partial_{\varepsilon}^{m_{2}} u_{p}\right)(t, z, \varepsilon) \\
& +\sum_{l=1}^{D-1} \sum_{m_{1}+m_{2}+m_{3}=m} \frac{m !}{m_{1} ! m_{2} ! m_{3} !} \partial_{\varepsilon}^{m_{1}}\left(\varepsilon^{\Delta_{l}}\right) t^{d_{l}} \sigma_{q, t}^{\delta_{l}} \\
& \times\left(\left(\partial_{\varepsilon}^{m_{2}} c_{l}\right)(t, z, \varepsilon) R_{l}\left(\partial_{z}\right)\left(\partial_{\varepsilon}^{m_{3}} u\right)(t, z, \varepsilon)\right)+\sigma_{q, t}\left(\partial_{\varepsilon}^{m} f\right)(t, z, \varepsilon)
\end{aligned}
$$

for all $m \geq 0$, all $(t, z, \varepsilon) \in \mathcal{T} \times H_{\beta^{\prime}} \times \mathcal{E}_{p}$. We let $\varepsilon$ tend to zero in the last equality (125) and use (124) in order to get the next recursion relation

$$
\begin{aligned}
Q\left(\partial_{z}\right) \sigma_{q, t} h_{m}(t, z) & \\
= & \frac{m !}{\left(m-d_{D}\right) !} t^{d_{D}} \sigma_{q, t}^{d_{D} / k+1} R_{D}\left(\partial_{z}\right) h_{m-d_{D}}(t, z) \\
& +\sum_{l=1}^{D-1} \sum_{m_{2}+m_{3}=m-\Delta_{l}} \frac{m !}{m_{2} ! m_{3} !} t^{d_{l}} \sigma_{q, t}^{\delta_{l}}\left(\left(\partial_{\varepsilon}^{m_{2}} c_{l}\right)(t, z, 0) R_{l}\left(\partial_{z}\right) h_{m_{3}}(t, z)\right) \\
& +\sigma_{q, t}\left(\partial_{\varepsilon}^{m} f\right)(t, z, 0)
\end{aligned}
$$

for all $m \geq \max _{l=1}^{D-1}\left\{d_{D}, \Delta_{l}\right\}$, all $(t, z) \in \mathcal{T} \times H_{\beta^{\prime}}$. For the reason that the functions $c_{l}(t, z, \varepsilon)$ and $f(t, z, \varepsilon)$ are analytic with respect to $\varepsilon$ at 0 , we see that

$$
c_{l}(t, z, \varepsilon)=\sum_{m \geq 0} \frac{\left(\partial_{\varepsilon}^{m} c_{l}\right)(t, z, 0)}{m !} \varepsilon^{m}, \quad f(t, z, \varepsilon)=\sum_{m \geq 0} \frac{\left(\partial_{\varepsilon}^{m} f\right)(t, z, 0)}{m !} \varepsilon^{m}
$$

for all $\varepsilon \in D\left(0, \varepsilon_{0}\right)$, all $z \in H_{\beta^{\prime}}, 1 \leq l \leq D-1$. On other hand, one can check by direct inspection from the recursion (126) and the expansions (127) that the formal series $\hat{u}(t, z, \varepsilon)=\sum_{m \geq 0} h_{m}(t, z) \varepsilon^{m} / m$ ! solves the equation (81). 


\section{References}

[1] Andrews, G. E., Askey, R. and Roy, R., Special functions, Encyclopedia of Mathematics and its Applications, 71, Cambridge University Press, Cambridge, 1999.

[2] Balser, W., From divergent power series to analytic functions. Theory and application of multisummable power series, Lecture Notes in Mathematics, 1582, Springer-Verlag, Berlin, 1994.

[ 3 ] Costin, O. and Tanveer, S., Short time existence and Borel summability in the Navier-Stokes equation in $\boldsymbol{R}^{3}$, Comm. Partial Differential Equations, 34 (2009), 785-817.

[4] Di Vizio, L., Ramis, J.-P., Sauloy, J. and Zhang, C., Équations aux $q$-différences, Gaz. Math., No. 96 (2003), 20-49.

[5] Di Vizio, L. and Zhang, C., On $q$-summation and confluence, Ann. Inst. Fourier (Grenoble), 59 (2009), 347-392.

[6] Dreyfus, T., Building meromorphic solutions of $q$-difference equations using a Borel-Laplace summation, Int. Math. Res. Not. IMRN, 2015 (2015), 6562-6587.

[7] Hsieh, P. and Sibuya, Y., Basic theory of ordinary differential equations, Universitext, Springer-Verlag, New York, 1999.

[8] Lastra, A. and Malek, S., On $q$-Gevrey asymptotics for singularly perturbed $q$-differencedifferential problems with an irregular singularity, Abstr. Appl. Anal., 2012 (2012), Art. ID 860716, $35 \mathrm{pp}$.

[9] Lastra, A. and Malek, S., On parametric Gevrey asymptotics for some nonlinear initial value Cauchy problems, J. Differential Equations, 259 (2015), 5220-5270.

[10] Lastra, A. and Malek, S., Parametric Gevrey asymptotics for singularly perturbed PDEs with delays, Abstr. Appl. Anal., 2013 (2013), Article ID 723040, 18pp.

[11] Lastra, A., Malek, S. and Sanz, J., On $q$-asymptotics for linear $q$-difference-differential equations with Fuchsian and irregular singularities, J. Differential Equations, 252 (2012), 5185-5216.

[12] Malek, S., On singularly perturbed $q$-difference-differential equations with irregular singularity, J. Dyn. Control Syst., 17 (2011), 243-271.

[13] Malek, S., On Gevrey asymptotics for some nonlinear integro-differential equations, J. Dyn. Control Syst., 16 (2010), 377-406.

[14] Marotte, F. and Zhang, C., Multisommabilité des séries entières solutions formelles d'une équation aux $q$-différences linéaire analytique, Ann. Inst. Fourier (Grenoble), 50 (2000), 1859-1890.

[15] Ramis, J-P., About the growth of entire functions solutions of linear algebraic $q$-difference equations, Ann. Fac. Sci. Toulouse Math. (6), 1 (1992), 53-94.

[16] Ramis, J-P., Sauloy, J. and Zhang, C., Local analytic classification of q-difference equations, Astérisque, No. 355, 2013.

[17] Ramis, J-P., Sauloy, J. and Zhang, C., Développement asymptotique et sommabilité des solutions des équations linéaires aux q-différences, C. R. Math. Acad. Sci. Paris, 342 (2006), $515-518$.

[18] Ramis, J-P. and Zhang, C., Développement asymptotique $q$-Gevrey et fonction thêta de Jacobi, C. R. Math. Acad. Sci. Paris, 335 (2002), 899-902.

[19] Tahara, H. and Yamazawa, H., $q$-analogue of summability of formal solutions of some linear $q$-difference differential equations, Opuscula Math., 35 (2015), 713-738.

[20] Tahara, H. and Yamazawa, H., Multisummability of formal solutions to the Cauchy problem for some linear partial differential equations, J. Differential Equations, 255 (2013), 3592-3637. 
[21] Yamazawa, H., Holomorphic and singular solutions of $q$-difference-differential equations of Briot-Bouquet type, Funkcial. Ekvac., 59 (2016), 185-197.

[22] Zhang, C., Transformations de $q$-Borel-Laplace au moyen de la fonction thêta de Jacobi, C. R. Acad. Sci. Paris Sér. I Math., 331 (2000), 31-34.

\author{
nuna adreso: \\ Laboratoire Paul Painlevé \\ University of Lille 1 \\ 59655 Villeneuve d'Ascq cedex \\ France \\ E-mail: Stephane.Malek@math.univ-lille1.fr
}

(Ricevita la 26-an de marto, 2015)

(Reviziita la 11-an de aŭgusto, 2015) 